\title{
Media Addiction Behaviour, Computer Vision Syndrome and Neural Patterns
}

Gahangir Hossain ( $\square$ gahangir@gmail.com )

West Texas A\&M University

Musab Faiyazuddin

Texas A\&M University-Kingsville

\section{Research Article}

Keywords: Media Interaction, Media Addiction Behavior, Brain Signal Analysis, Computer Vision Syndrome

Posted Date: March 23rd, 2022

DOI: https://doi.org/10.21203/rs.3.rs-1292985/v2

License: (c) (1) This work is licensed under a Creative Commons Attribution 4.0 International License. Read Full License 


\section{Abstract}

These Post-COVID days, seventy percent of businesses have or are working on a digital transformation, which make us to spend more time with media interaction [1]. Media has always existed in much of our history; the shape and function of such media instruments may have evolved over time, but human nature has always been drawn to them. Social networking is one of the most common and addictive media resources available today. They have only been around for a decade, but they're more appealing and addictive in nature. According to a recent survey, there are approximately 400 million social media users, and 5 to $10 \%$ of the world's population suffers from gaming or internet addiction [2]. Addiction is described as a person's neurotic behavior that contributes to harmful behavior - social media addiction cannot be the exception. This research explores human brain signals, electroencephalogram (EEG) of addictive media abusers and compare it to that of alcoholics to draw a distinction between these two different types of addictions. We performed brain signal analysis in rest stage and working stages. The magnitude and spatial distribution of beta power in the resting EEG were examined to address the possibility of an excitation-inhibition imbalance in the central nervous system of alcoholics with compared to medica addiction. Similar increase in Beta absolute power was observed in alcohol dependent as well as media addictive subjects. In addition, we observed that human eye blink rates that reflects human attention, also reflects media addiction - especially the Computer Vision Syndrome (CVS). While performing attentive tasks on digital screens causes a decrease in eye blink rate (EBR), which leads to the fundamental symptom of eye strain or the CVS.

\section{Introduction}

Media has always existed for much of our history; the shape and content of such media may have evolved over time, but the media resources have always attracted human nature. One of the most influential and addictive media resources of our time is social media [1]. They've only been around for a decade, but they're more appealing and addictive in nature. According to a recent survey, there are approximately 400 million social media users [1]. Using social media, such as Facebook, has grown over time and has expanded beyond just connecting with friends to include social networks, ads, and marketing. This form of continuous evolution may be one of the key explanations for people's connection to them.

Social media wields such control and influence over the public that it has surpassed the use of traditional media technologies such as television and newspapers. This begs the question, "What is social media's addictive nature?" 'And what do the public see on social media?' 'Is the time we spend on social media worthwhile?' is the other side of the debate.

Addiction is a condition under which a person's compulsive conduct results in negative consequences. People are usually so drawn to such behaviors that they become a habit, an addiction that disrupts other aspects of their lives [3]. So, is it possible that there is such a thing as an "internet addiction"? The response is, in a nutshell, "Yes." Human psychology, according to studies, is largely self-aware. When our 
brain perceives something as a reward, it produces dopamine, making us feel good [4]. The use of social media, with its constant desire for likes and feedback, can cause this release of dopamine to occur more frequently.

In 2009, a boy in South Korea died of malnutrition. The parents of the three-year-old were found to be regularly spending 12-hour gaming sessions in the online world, according to the investigation. This was the first court case in South Korea dealing with gaming addiction, and the child's mother was taken into custody due to her addiction. Despite the fact that this is an especially heartbreaking tale due to the death of an innocent child [8]. People shirking their duties, their wellbeing, and even their lives are involved in a surprising number of cases involving video games. There have been several reports of men found dead on their computers in Asian internet cafes [9]. They would always play for hours on end with no food, water, or sleep. It's a common feeling in a world where internet addiction has become a public health problem. Teenage security systems are falling behind as technology becomes more ubiquitous.

The approach used in the study is depicted in Fig. 1. The information we gathered is initially unprocessed. Filtering methods are used to remove noise and artifacts from results. After that, we look for neural markers and trends in the filtered results. We draw conclusions and compare similar data for different types of addictions based on the neural data values.

\section{Computer Vision Syndrome (CSV)}

Long-term use of computer screens, tablets, e-readers, and mobile phones-Video Display Terminals (VDT)-has been related to vision problems affecting more than 60 million people around the world [10]. The Computer Vision Syndrome (CVS) [10] is a name for a group of computer-related eye problems. CVS is bidirectional in that it affects individuals differently depending on their symptoms. The study also supported our hypothesis that if eye blink rate variability (BRV) is a reasonable predictor of human attentiveness, then human attention can be directly linked to CVS. Two EEG signals were analyzed, one from the participant while performing a difficult cognitive task and the other from a control subject.

In today's technology-driven world, using Digital Electronic Screens or Visual Display Terminals (VDT) is virtually impossible to do without [11]. For knowledge, entertainment, interaction, and education, among other things, people have never been more reliant on television, computers, mobile phones, and tablets [11]. Part of the reason for the shift from typewritten hard copy materials and other acceptable alternatives to these digital devices was their now lower purchasing and operating costs, as well as their ready availability, user-friendliness, and portability. The invention and marketing of the Internet, which is based on electronic platforms, has also had a huge impact.

Despite the conveniences and customizability of electronic devices, excessive usage of electronic devices has been linked to negative health effects in users [11]. Users tend to experience eye and visual symptoms after extended use. These eye and visual symptoms are usually referred to as DES (Digital Eye Strain) or CVS (Computer Vision Syndrome) [13], with the first being favoured by some since not all VDT computers are available to the general public. CVS is characterized by a variety of symptoms and is 
multidirectional in nature, as its impact varies with age, sex, and visual problems. Eye pain, headaches, and blurred vision close and far are the most common CVS symptoms [13].

We go through the signs of Computer Vision Syndrome and how eye blink rate is used as an indicator, as well as analyzing two sets of EEG signals to see how focus affects eye blinks. The approach is divided into two parts (see Fig. 2.), each with two primary objectives. The first goal would be to determine if the variability of the blink rate is a valid method of measuring human attention by analyzing two EEG data sets. Blink rate data is extracted from EEG data sets in the second step. The next step will be to use Independent Component Analysis to extract objects from the results (ICA).

\section{Background}

\subsection{Electroencephalography (EEG)}

Electroencephalography, or EEG for short, is a bio signaling technique that records electrical potential in brain cortex activities in real time to represent brain status [14]. EEG is one of many electrobiological measuring techniques, including ECG (Electrocardiography) for pulse measurement, EMG (Electromyography) for muscle contraction measurement, EOG (Electro-Optigraphy) for eye-dipole field measurement, and many other imaging methods based on bodily concepts, such as MRI (Magnetic Resonance Imaging) and CT-Scan (Computer Tomography) [14]. EEG is a type of electrical impulses measured by a conductor or meta on the scalp surface of the head.

The neutron summation of electrical events is included in the EEG [14]. Neutrons are excitable cells with unique intrinsic electrical properties that generate both electrical and magnetic fields when they interact. Electrodes are used to map these fields at a short distance from the cortical surface. The human brain is divided into three parts from an anatomical standpoint: the cerebrum, cerebellum, and brain stem. The brain is the most anterior and primary component of the human body; its surface is known as the cerebral cortex, and it is made up of the left and right hemispheres. The electrical impulses of the brain are tracked by EEG electrodes [15]. The cerebrum is an essential component of the central nervous system (CNS), and it is responsible for feeling, complex analysis, emotion, and other functions [15]. The brain stem oversees heartbeat, hormone synthesis, and respiratory rate, while the cerebellum maintains equilibrium and voluntary muscle activity [15]. The different electrode scalp positions are defined using two globally accepted nomenclatures. First, The naming scheme of 10-20 [16]: The 10-20 name was derived from the spacing of electrodes from each other with either $10-20 \%$ front-to-back electrode spacing or $10-20 \%$ left-to-right electrode spacing; the 10-20 name was derived from the spacing of electrodes with either $10-20 \%$ front-to-back electrode spacing or $10-20 \%$ left-to-right electrode spacing. Each electrode is designated by an alphabet and a number. F stands for Frontal Cortex, $\mathrm{T}$ for Temporal Cortex, and $\mathrm{C}$ for Central Cortex (It has no real purpose other than to identify). Similarly, P stands for the parietal region, and $\mathrm{O}$ stands for the occipital region. Positions on the left hemisphere of the head are denoted by odd numbers, while positions on the right side are denoted by even numbers. Second, The Modified Combinatorial Nomenclature [16] consists of the following terms: When more electrodes were needed for 
more reliable recordings, the need for this arose. The intermediate points in the 10-20 naming scheme are used in this system to link the different electrode locations. FC denotes the point halfway between $\mathrm{F}$ and C. T7, T8, P7, P8 stands for T3, T4, T5, T6 in the 10-20 modified combinatorial nomenclature; TP stands for the intermediate position between $\mathrm{T}$ and $\mathrm{P} ; \mathrm{CP}$ stands for the intermediate location between $\mathrm{C}$ and $\mathrm{P} ; \mathrm{PO}$ stands for the intermediate location between $\mathrm{P}$ and $\mathrm{O} ; \mathrm{T} 7, \mathrm{~T} 8, \mathrm{P} 7, \mathrm{P} 8$ stands for T3, T4, T5, T6 in the 10-20 modified combinatorial nomenclature.

EEG is one of the most difficult bio signals to interpret because of its low amplitude, which ranges from $0.5 \mathrm{~V}$ to $100 \mathrm{~V}$. It does, however, have a major speed advantage since complex brain patterns can be recorded in real time. Multiple neutron potential aggregation is what EEG waves are made up of. Human behavior is depicted by these frequency bands. The gamma waves, which display alertness, wakefulness, and anxiety, have a lower amplitude than the high-frequency beta waves. The amplitude of an alpha wave is lower than that of a beta wave, indicating that the individual is awake, calm, and learning. Theta waves signify rapid eye movement sleep and mindfulness, while delta waves are the slowest and have the largest amplitude, indicating $\mathrm{N}$.

\subsection{EEG Waves and Types}

Based on the brain wave frequencies (in Hz), EEG are classified as delta, theta, aloha and beta (Fig. 3).

Delta: Delta is equal to or less than $3 \mathrm{~Hz}$. In terms of amplitude, it happens to be the strongest and slowest waves. As the dominant rhythm in infants up to one year old, as well as in sleep stages 3 and 4 , it is completely normal. It may happen in a focal way with subcortical lesions and more often with diffuse lesions, hydrocephalus metabolic encephalopathy, or deep midline lesions. It is typically most noticeable frontally in adults (for example, FIRDA - Frontal Intermittent Rhythmic Delta) and then later in children (for example, OIRDA - Intermittent Rhythmic Delta Occipital) [17].

Theta: Theta has a frequency of 3.5 to $7.5 \mathrm{~Hz}$ and is considered "slow." It is completely common in children under the age of 13 while they are sleeping, but it is dangerous in awake adults. It is a symptom of focal sub-cortical lesions; diseases such as metabolic Encephalitic or certain forms of Hydrocephalus can also be seen in a wide range of people [17].

Alpha: Alpha has a frequency range of 7.5 to $13 \mathrm{~Hz}$. Is normally better seen in the back of the head on either side, with the dominant side's amplitude being higher. When the eyes are closed and comfortable, it appears. When some mechanism opens the eyes or warns, it vanishes. It's the most common rhythm in healthy adults who are comfortable. It is present for the majority of one's life after the thirteenth year [17].

Beta: Beta has 14 and higher $\mathrm{Hz}$ frequencies. Both sides are distributed symmetrically and is most obvious on the front. Sedative - hypnotic drugs, especially benzodiazepines and barbiturates, accentuate it. In neuronal damage locations, it may be missing or reduced. It is generally regarded as a steady rhythm. It is the dominant for patients that are alert and have their eyes open [17]

\subsection{EEG Recording}


Electrodes are small metal disks that are normally made of stainless steel and protected with a silver chloride coating. They are put on the scalp in specific places. These positions are specified by the International 10-20 framework [16]. A letter post with a number is fixed to each electrode spot. The alphabet is related to the brain (For Example, F- Frontal lobe). The right side of the head is represented by even numerals, while the left side is represented by odd numerals. The electrodes of the disks are added to the electrode gel and applied to the subject's scalp using EEG cables. A cap with electrodes encapsulated in it is used in many technologies to enable recording when electrode arrays are present.

Electrode Gel: Electrode gel serves as a malleable extension of the electrode, preventing objects from being generated by the electrode cables' movement. It optimizes direct communication and allows for the recording of low resistance. The electrolytic gel is placed in each cavity until a small amount of the hole in the mount is released. With a small amount of downward pressure, the syringe is easily rocked back and forth.

Electrode Positioning: We're adapting the 10/20 electrode positioning system from around the world. It's been very popular to provide a standardized positioning of electrodes for a classic EEG since the 10-20 arrangement was adopted (Fig. 4). The distance between Nasion Inion and other fixed points in percentages in the 10-20 range [16] is the essence of this scheme. Fp, C, P, O, and T are the names given to these points. The midline electrodes are indicated by a subscript ' $z$ '. Points to the left as a subscription are represented by odd numerals, while points to the right are represented by evens.

EEG Montages: Montages are rational and orderly channel arrangements [16]. A referential or bi-polar installation may be used to observe the EEG. Each channel in bipolar has a reference electrode [16]. Both channels would have a shared reference thanks to the referential assembly.

Artifacts: Recognizing and eliminating artifacts is a big challenge in EEG tracking [17]. There are subjectrelated artifacts (for example, eye or muscle movements) as well as technical artifacts (for example, static caused by cable movements) [17] that must be handled differently. There are several resources that can be used to locate the objects. Measurements of FEMG and impedance, for example, may be used to detect a polluted signal [17]. Another source of interference can be discovered by examining different variables on a monitor. The electrodes used in EEG recording do not discriminate against the electrical signals they receive [17].

An artifact is any documented behavior that is not of cerebral origin. It is divided into physiological activity (activity produced by the subject other than the brain) and extra physiological objects (equipment such as electrodes and environment) that originate from outside the body.

Eye movement: With a cornea and a retina (+ and - poles, respectively), the eyeball serves as a dipole [16]. A broad amplitude alternating current field is produced as the globe rotates around its axis, which can be detected by any of the near-eye electrodes. The cornea (+ Pole) moves closer to the two frontopolar electrodes while the eye is blinking [17].

\subsection{Role of Eye Blinks}


Blinking is one of several identified human physiological functions [18]. It is best described as the involuntary rapid closing of the eyelids. It has also been discovered to play a key role in high-order cognitive efficiency, in addition to its protection feature of shielding the eye from potentially harmful external contamination and drying. The Central Nervous System (CNS) is said to be in charge of controlling eye blinking [18], with dopamine being the main neurotransmitter involved, especially in the Globus pallidus region. In terms of higher order functioning, blinking and its frequency have also been linked to consciousness, intelligence, and overall day-to-day functioning. In a clinical research study conducted by SA Chermahini and B Hommel, it was discovered that eye blink rate (EBR) was negatively correlated with convergent thought ('Remote Association Task') and had a positive relationship with intelligence [19]. Although they discovered that divergent thinking was strongly linked to average EBR [19], they also discovered that divergent thinking was strongly linked to average EBR.

\subsection{Addictions}

Addiction is described as a state of compulsive desire [3]. It continues to relapse after therapy and other efforts to control drug use. Addiction is the malignant abdication of neuronal pathways that usually serve to reward, according to medical and research facilities [3]. Almost all addictions cause the neurotransmitter dopamine to be released in excess and at irregular levels, affecting the Limbic System of the brain (Rewarding). Chronic opioid and alcohol users have destroyed receptors in the brain for dopamine and other main neurotransmitters, which must be restored during addiction recovery. Furthermore, each narcotic drug has a distinct negative impact on the brain. How can one assess the brain's addiction to internet media? Behavioral addictions, including opioid addictions, disrupt and pervert reward processes in the brain. Impulsivity modulation is also linked to behavioral addictions. Changes in prefrontal cortex activation and frontal striatal pathway activation were seen in FMRI internet addiction studies.

\section{Methodologies}

The research methods were carried out in accordance with relevant guidelines and regulations. The data set including the protocols was passed through the IRB at the Texas A\&M University-Kingsville, TX for secondary analysis. The experimental protocol was approved by Dr. Rafael Paprocki's Korea University of Technology and Education [18], which was generously donated for secondary analysis. Informed consent was obtained from all subjects and/or their legal guardian(s) by the primary source of identifying information/images. The dataset used and analyzed during the current study is available from Dr. Rafael Paprocki on reasonable request [18].

\subsection{Participents}

In his research, he enlisted the help of 51 healthy volunteers [18]. The EEG signals were collected using a wearable multi-band interface (Emotiv Epoc + EEG). With 14 electrodes located in the 10-20 normal positions, the unit has 8 channels and 2 reference channels. (See Fig. 5) During the experiments, the participants sat in a comfortable seat that was $65-70 \mathrm{~cm}$ away from a 15 -inch display screen. 


\subsection{Methods and data}

Participants wore a 14-electrode EEG cap with eight data channels in the international 10-to-20 scheme. The participants were divided into two classes in general. 1. People who spend a lot of time on social media, games, and screens in general. 2. Those that aren't seen as much. After the data was recorded, it went through a filtering process that included 1. High-pass Filtering and 2. Bandpass Filtering, among other things. For each channel, various plots and spectrograms were created using the filtered spectrum data. Working with this data can be difficult at times, so we use a variety of tools and packages to help. The following is an example:

EEGRUNT [20]: EEGRUNT is an Open Source library designed to make neural data analysis more straightforward. It entails a set of techniques for reading EEG data from CSV files, cleaning it in various ways, and finally generating visually appealing and informative visualizations. This method will be used to analyze data and create plots for visualizations.

MATPLOTLIB [21]: For data refining and visualization, we'll use Python's software and packages. MATPLOTLIB is one of them. PYPLOT is a Python plotting program. It is a collection of command-style functions that allow us to use MATLAB's features. Any other pyplot function, for example, produces a figure, assists in the creation of a plotting field, plots lines in it, labels the figure, and so on.

EEGLAB [22] (see in Fig. 6) is a graphical toolbox for continuous and event-related EEG, MEG, and EOG processing, as well as other electrophysiological data, that incorporates ICA (Independent Components Analysis), time frequency analysis, anomaly dismissal, event-related stats, and many other useful simulation methods for average and single trial data.

It also has an interactive graphical interface that allows the user to process their EEG and other vibrant brain data using ICA (Independent Component Analysis), TFA, and regular average processes in a flexible and interactive manner. It also has robust help and support windows, as well as a commands history feature that makes the transition from GUI to batch or custom analytics scripts easier. EEGLAB offers a range of strategies for visualizing and tagging event-related central nervous system mechanics, both at the data set level and through a series of datasets gathered in EEGLAB.

EEGLAB is a coding environment that allows experienced MATLAB users to store, analyze, modify, and imagine EEG proof related to events. EEGLAB provides an expandable open-source forum for programmers and developers of novel data collection methods to encourage new techniques with the international research community by publishing 'plugin' functions that appear in the menu. New plugins could be created and released to 'pick spikes in ERP view or TF performance, visualization, or reverse source designing of EEG, MEG, or EOG data, for example.

ERPs (Event-Related Potentials) [16]: ERPs are a non-invasive instrument for calculating neural activity during cognitive function. The components of ERP (temporary changes in electrical potential) are locked to the start of the stimulus. Each of these elements represents a brain function involving one or more 
neuronal activities. ERPs are characterized by multidimensional online voltage (+ or - potential), frequency, throughput, and scalp distribution all at the same time, unlike behavioral measures such as error margins and reaction times. ERPs may thus be used to identify and distinguish neurological and cognitive sub-mechanisms involved in higher cognitive and auditory tasks.

The additional benefits of ERPs also led to a growing number of ERP experiments in neuropsychology, personality psychology, linguistic anthropology, neurobiology, and neuroscience.

i. Granger Causality is a method of examining relativity in a data series involving two variables. The method is a quantitative account of causality that employs analytical data sets to find patterns of association. Even if they are not the same thing, causality is inextricably related to the cause-andeffect concept. A vector $A$ is the cause of $B$ if $A$ is the source of $B$ or $B$ is the cause of $A$. Even so, Granger causality does not measure a true cause-and-effect relationship; what you want to know is whether one variable comes before another in the time chain. To put it another way, if you find Granger causality in your results, there is no conclusive conclusion. Granger causality is a "bottomup" methodology in which the data-generating functions in each data series are independent variables, and then the data sets are evaluated to see whether they are strongly related. The inverse is a "top down" technique in which the procedures are assumed to be non-autonomous, and then the data sets are analyzed to see whether they are generated independently of one another. The method can become complicated due to the large number of options available, such as choosing from a collection of mathematical models for f-value analyses. Most extra measures can be avoided by using apps. Granger's causality test is used in several powerful economics software packages. ICA [22]: Independent Component Analysis is a common quantitative tool for separating multivariate scalp nerve impulses into self-reliant additive or source components, such as electroencephalograms and pattern extraction (see Fig. 7). A directional shift from data collected on scalp channels to a temporally modified "virtual channel" basis is included in the decomposition of ICA data. In other words, instead of collecting single channel data simultaneously, the data is transformed into a series of concurrently registered temporal filter outputs that are applied to all multi-channel data. The original scalp channel data represents $t$ in each row of the data recording matrix.There are many ICA implementation algorithms and methods. We used the FastlCA algorithm in this research. In the following flowchart, it could be summarized and explained:

FastICA is an efficient and widely used Independent Component Analysis algorithm created by Aapo Hyvärinen at the University of Technology in Helsinki [23]. FastICA, like many other algorithms, aims to rotate pre-whitened data orthogonally using a set-point iteration scheme that maximizes non-gaussian rotational component calculation. Non-Gaussianity offers a loophole for quantitative separation, a very strong requirement that necessitates the verification of infinite results. FastICA can also be derived as an approximate Newton iteration [23]. Flowchart for FastlCA Algorithm is shown in Fig. 8.

\section{Data Analysis And Results}


Independent Component Analysis: Here we adapted the FastICA algorithm to implement ICA in our research as mentioned in the chapters before. It extracted the following component maps for a summary view of the activity power spectrum and event - related data epochs. The 2D component map is shown in Fig. 9.

To plot maps of 2-D scalp components, select Plot -> Component maps -> In 2D. Pop topoplot.m will then generate the animated window. The popup window we used to plot ERP scalp maps is very similar.

Blinks and artifacts identification: Fig. 10. Shows IC1 representing blinks in the spectrum.

For three factors, this Fig. 10 can be recognized as an eye artifact: (1) A typical eye artifact has the smoothly decreasing EEG spectrum like the one displayed; (2) The scalp shows a powerful, far - front visualization typical that of an eye artifact; And, (3) Individual eye movements can be seen in the erpimage.m aspect

Eye objects are almost always present in EEG datasets. They are usually found near the top of the collection of components and contour maps of the scalp. Component property figures can also be accessed directly by choosing Plot $\rightarrow$ Component properties. The single component 1 is shown in Fig. 10 with a clear frontal eye movement projection. Several eye blinks were saved because they're one of the most important parts of our research. Most of the objects are dismissed or decayed based on the severity of their errors. Individual data components with blinking elements (see Fig. 11) and cross coherence (see Fig. 12).

One EEG was collected while the individual was engaged in a challenging cognitive task and compared to a control data set collected when the person was in a less reflective role. The blinking rate of subjects performing cognitive tasks decreased significantly from 19 to 15 in signals obtained from them. After counting the blinks in our data sets, we noticed a strong difference between the blinks caused by a more attentive task and the blinks caused by a less attentive task. On the power spectral density estimates, it can also be seen that power is most prevalent in the alpha frequency region and gradually decreases as the frequency increases. Furthermore, the spectral analysis is useful.

An Event-Related Potentials (ERP) is also analyzed (see Fig. 13). From the above results, it can therefore be deduced that blinking rates decrease when conducting a conscientious task on Video Display Terminals (VDT), which will help spur eyestrain - a major symptom of CVS. High attention in the use of VDT is therefore directly linked with CVS. The component Activity IC14 and Standard Normal Quantities is shown in Fig. 14. Finally the Power spectral density for components are shown in Fig. 15.

\section{Power Spectral density}

Average power of a signal $x(t)$ for the total time is given by the succeeding time average 


$$
P=\lim _{T \rightarrow \infty} \frac{1}{T} \int_{0}^{T}|x(t)|^{2} d t
$$

\section{EEG data and alcoholism}

Colrain analyzed 42 long-term alcoholics ( 27 males) and 42 controls (19 males) in a clinical study in 2009 [24]. According to Nicholas and Trinder's study [25], alcoholics were significantly less likely than controls to produce K-complexes. Despite this, the amplitude of P2 was lower in abusers than in controls, with the largest difference in Cz, alcoholics' P2 latency was longer [25]. There were no gender differences or relationships between screening and sex for K-complex occurrence, P2 amplitude, or P2 latency. Heavy drinkers had lower frontal amplitudes of N550 and P900 than controls, and males had lower frontal amplitudes than females, but the gender difference was not important ( see Fig. 16).

As a result, this research shows that long-term alcohol dependence is linked to a reduced capacity to produce evoked delta waveform responses as well as a smaller response order of magnitude when evoked (weaker N550 and P900 amplitudes). The resting EEG is a transcript of current nervous system electrical impulses recorded while the subject is in a relaxed state. It is made up of oscillations with a frequency defined as the number of times a wave completes its loop in one unit of time. By the magnitude of their voltage, EEG variations are often calculated in microvolts $(V)$ (power).

EEGs are typically classified into five frequencies: Delta (3Hz or less), Theta $(3.5-7.5 \mathrm{~Hz})$, Alpha $(8.0-11.5$ $\mathrm{Hz}$ ), Beta (between 12 and $28 \mathrm{~Hz}$ ), and Gamma (between 28.5 and $50.0 \mathrm{~Hz}$ ), with each frequency indicating a different degree of cognitive activity. Via safe adult lives, the EEG remains stable and highly heritable [26]. Alcoholics have irregular resting EEGs and ERPs, according to this study. Often, explore the significance of the findings. Tonic theta levels have been shown to rise in many brain conditions, including Alzheimer's disease, and to rise with reduced brain activity, according to neuroscientists. The Collaborative Study on Alcohol Genetics (COGA) looked into alcoholics' tonic theta capacity. The incidence of eye-closed resting theta in 307 people who participated in the alcohol-dependent survey and 307 people who were matched by sex in a study [27]. The alcohol-dependent group had higher resting theta in all scalp locations. Increased tonic theta intensity in the EEG can suggest a problem with cognition ability in the Central Nervous System (CNS) [28]. Theta capacity at rest increased with age, and also in Alzheimer's patients [28]. Another possible rise in theta may be a neurophysiological sign of a cortical imbalance in the balance of excitatory and inhibitory neurons [28].

In a relaxed human, the alpha rhythm is the highest of all frequencies. It can be seen when the eyes are open or closed, and it is greatest over the occipital region of the brain when the eyes are closed. The alpha frequency is a calming factor. Several major studies, dating back to the mid-19th century, have shown that alcohol abusers have unbalanced or low alpha capacity. Initial research suggested that addicts had lower alpha capacity than non-addicts [30], but more recent research contradicts this [29]. Enoch and his team discovered that an alpha rhythm, specifically Low Voltage Alpha (LVA. T), was linked 
to an alcohol dependence co-subtype occurring with social anxiety. The authors believe that the effect of altered NE levels on thalamus stimulation (a nervous system messaging facility) can explain some of the links between LVA, anxiety, and alcoholism. (a nervous system messaging system) may clarify some of the connections between LVA, anxiety, and alcoholism. [23.]

EEG data and Media Addicts:

When it comes to Media Obsessed people, we've done a lot of electroencephalogram data analysis. We discovered some strong findings about theta and alpha frequencies of addicts as compared to nonaddicts. Since addict research has suggested that abusers have low alpha and high theta, the same has been applied to the measurement of all subjects' median alpha and theta frequency bands. In the occipital region of addicted subjects, a whole pattern in alpha and theta signals was discovered to be dominant and peculiar compared to the other frequencies. We may infer from other analytics we performed, such as eye blink rate and human attention, that performing attentive tasks on digital screens causes a decrease in eye blink rate (EBR), which leads to the fundamental symptom of eye strain computer vision syndrome (CVS). Overall trend of Alpha \& Theta of Media Addicted vs non-Addicted are shown in Fig. 17. The same data also can be explained through the Table 1. It is observed that addicted signals are always higher frequencies (in $\mathrm{Hz}$ ) than the normal behavior.

Table 1. Table showing absolute Delta, Alpha and Theta at each electrode position

\begin{tabular}{|c|c|c|c|c|c|c|}
\hline Electrode & \multicolumn{2}{|c|}{ Absolute Delta } & \multicolumn{2}{c|}{ Absolute Theta } & \multicolumn{2}{c|}{ Absolute Alpha } \\
\hline & Addicts & Normal & Addicts & Normal & Addicts & Normal \\
\hline T3 & 130.40 & 104.40 & 109.50 & 93.50 & 52.00 & 48.60 \\
\hline T5 & 150.20 & 144.40 & 156.30 & 144.70 & 88.20 & 98.30 \\
\hline F3 & 180.40 & 152.40 & 162.20 & 140.80 & 72.20 & 66.70 \\
\hline C3 & 179.80 & 151.03 & 165.80 & 145.70 & 85.70 & 79.40 \\
\hline P3 & 182.90 & 162.50 & 191.40 & 175.20 & 116.10 & 110.80 \\
\hline O1 & 202.30 & 191.30 & 224.50 & 205.40 & 170.50 & 197.70 \\
\hline T4 & 134.80 & 104.30 & 115.10 & 93.40 & 57.50 & 47.50 \\
\hline T6 & 175.40 & 142.90 & 181.40 & 150.90 & 131.80 & 105.70 \\
\hline F4 & 187.90 & 152.90 & 166.60 & 143.30 & 71.20 & 67.30 \\
\hline C4 & 182.00 & 155.50 & 168.40 & 149.70 & 88.50 & 83.10 \\
\hline P4 & 198.40 & 172.00 & 194.30 & 175.40 & 124.50 & 110.56 \\
\hline O2 & 220.50 & 191.40 & 230.30 & 210.50 & 194.30 & 202.30 \\
\hline F0 & 201.20 & 165.01 & 191.10 & 160.30 & 77.20 & 71.30 \\
\hline C0 & 218.99 & 185.40 & 202.12 & 190.50 & 94.10 & 89.90 \\
\hline P0 & 211.30 & 184.20 & 211.40 & 195.70 & 124.80 & 118.70 \\
\hline
\end{tabular}

\section{Power Spectrum Analysis:}

We determined the spectral density to consider the power signal distribution in the frequency domain. To get such, the Fourier transform was used. We discovered that the power of addicts was significantly 
higher than that of non-addicts, especially in the occipital region of the brain. Even though the power was higher in all areas of the brain, the occipital region showed a noticeable difference. The spectrograms and power spectral analysis are shown in the Fig. 18-20. Power spectrum for channel 8 to channel 1 are shown in Fig. 18, the Trend graph are shown in Fig. 19, and Spectrograms are shown in Fig. 20.

We used Python's matplot.lib and MATLAB's EEGLAB to plot the power spectrums, trend graphs, and spectrograms. We calculated the absolute values for the frequency bands Alpha, Theta, and Beta of the EEGs in Table 1 using these. The overall pattern of Alpha and Theta was found to be similar to that of alcoholics. When digital displays are removed from the equation, the results appear to change.

\section{Conclusion And Future Scope}

The aim of this study was to look at neural trends in EEG data from people who are addicted to social media and video games. Furthermore, we conducted a comparative analysis with previous studies on subjects addicted to alcohol and discovered a similar baseline between the two addictions. We expected that most alcoholics' EEG data will coincide with that of media addicts. We also discovered that with Media Addiction, which necessitates prolonged use of digital displays, the average eye blink rate decreased over time, resulting in eye pressure, which is one of the most common symptoms of Computer Vision Syndrome.

For further applications of this study, advances in technology such as headsets or watches that can monitor addiction tendencies might be available soon. There have been several developments in the applications of the most recent mobile phones that are geared toward the users' Digital Wellbeing. However, much more research and development are needed in this field to stop media addiction before it progresses. Future research in the field of Computer Vision Syndrome is also required to solve the human ocular problem caused by digital displays.

\section{Declarations}

\section{Conflict of interest disclosures:}

This work has been performed in Texas A\&M University-Kingsville. This work was not funded from any sources. Author Musab Faiyazuddin, and Gahangir Hossain declare that they have no conflict of interest.

\section{References}

1. Myrian $\mathrm{H}$ and Vivian A, "The Impact of Social Media in the Workplace", Proceedings of the 11th Annual College of Education \& GSN Research, January 2012.

2. Karim R and Chaudhri P, "Behavioral addictions: an overview”, J Psychoactive. Drugs, March 2012.

3. Steve S and Alan N.S, "Considering the Definition of Addiction", Int. J. Environ. Res. Public Health, October 2011. 
4. Richard M.R and Edward L.D, "Self-Determination Theory and the Facilitation of Intrinsic Motivation, Social Development, and Well-Being”, American Psychological Association, January 2000.

5. Esteban P, Alvaro M.P and Amalia L, "Visual and ocular effects from the use of flat-panel displays", International Journal of Ophthalmology, June 2016.

6. Arnaud D and Scott M, "EEGLAB: an open source toolbox for analysis of single-trial EEG dynamics including independent component analysis", Journal of Neuroscience Methods, October 2003

7. Javier L.C and Steven J.L, "ERPLAB: An open-source toolbox for the analysis of event-related potentials", Frontiers in Human Neuroscience, April 2014

8. Tony B, "Computer Game Addict Left Son, 2, to Die so That He could Play I The Times." The Times, April 2014.

9. Katie H, "Man dies in Taiwan after 3-day online gaming binge I CNN" CNN Jan, 2015.

10. Akinbinu T.R and Mashalla Y.J, "Impact of Computer technology on health: Computer Vision Syndrome (CVS)" Academic Journals, November 2014.

11. Leonid M, "The impact of smartphones and mobile devices on human health and life", American Journal of Education Research, Vol 5, 2017.

12. Mary M, and Sydney J, "Networked workers", Washington, DC: Pew Internet \& American Life Project, September 2008.

13. Camelia M.B, Diana E.S and Corina A.N. "Eyesight quality and Computer Vision Syndrome”. Romanian Journal of Ophthalmology, April-June 2017.

14. Mitsuru T, Manabu G and Koji I, "Fundamental Research about Electroencephalogram (EEG) Functional Electrical Stimulation (FES) Rehabilitation System", IEEE 11th International Conference, Japan, June 2009.

15. P. Thomas S, "Evolution of the Size and Functional Areas of the Human Brain" Annual Review of Anthropology, June 2006.

16. Ernst $\mathrm{N}$ and Fernando L.D, "Electroencephalography: Basic Principles, Clinical Applications, and Related Fields", Lippincott Williams \& Wilkins, 2011.

17. Ivan M.B, "EEG Artifact Detection”, Department of Cybernetics, Czech Technical University in Prague, June 2010.

18. Rafal P, Temesgen G, Marija G and Artem L, "Extracting Blink rate variability from EEG signals" arXiV, April 2016.

19. Chermahini SA and Hommel B, "The (b)link between creativity and dopamine: Spontaneous eye blink rates predict and dissociate divergent and convergent thinking", Cognition Elsevier, June 2010.

20. Arzu G and H. Levent A, "An SSVEP Based BCl to Control a Humanoid Robot by Using Portable EEG Device", 35th Annual International Conference of the IEEE EMBS Osaka, July 2013.

21. Paul B, John H, Todd M, Jin-Chung H and Perry G, "Matplotlib: A Portable Python Plotting Package" Astronomical Data Analysis Software and Systems, vol. 142004. 
22. Delorme A and Makeig S, "EEGLAB: an open source toolbox for analysis of single-trial EEG dynamics including independent component analysis," Journal of Neuroscience Methods, vol. 134, March 2004.

23. Hyvärinen A, "Fast ICA for noisy data using Gaussian moments", Proceedings ISCAS, vol. 5, May June 1999.

24. Ian M.C, Sharon T, Fiona C.B, "Impact of alcoholism on sleep architecture and EEG power spectra in men and women", SLEEP, October 2009.

25. Christian L.N, John T, and lan M.C, "Increased production of evoked and spontaneous K-complexes following a night of fragmented sleep." SLEEP, July 2002.

26. C.E. Beijsterveldt, P.C. Molenaar, E.J. de Geus and D.I. Boomsma. "Heritability of human brain functioning as assessed by electroencephalography", The American Society of Human Genetics, July 1995.

27. Kamarajan C, Porjesz B and Jones KA, "Alcoholism is a disinhibitory disorder: neurophysiological evidence from a go/no-gotask", Biol Psychol, July 2005.

28. Klimesch W, Doppelmayr M, Wimmer H, Schwaiger J, Rohm D, Gruber W and Hutzler F, "Theta band power changes in normal and dyslexic children", Clinical Neurophysiol, July 2001.

29. Enoch M.A, White KV, Harris CR, Robin RW, Ross J, Rohrbaugh JW, "Association of low-voltage alpha EEG with a subtype of alcohol use disorders", Alcohol Clin Exp Res, August 1999.

30. Porjesz B, Begleiter H, and Samuelly I, "Cognitive deficits in chronic alcoholics and elderly subjects assessed by evoked brain potentials", Acta Psychiatrica Scandinavica, October 1980.

\section{Figures}




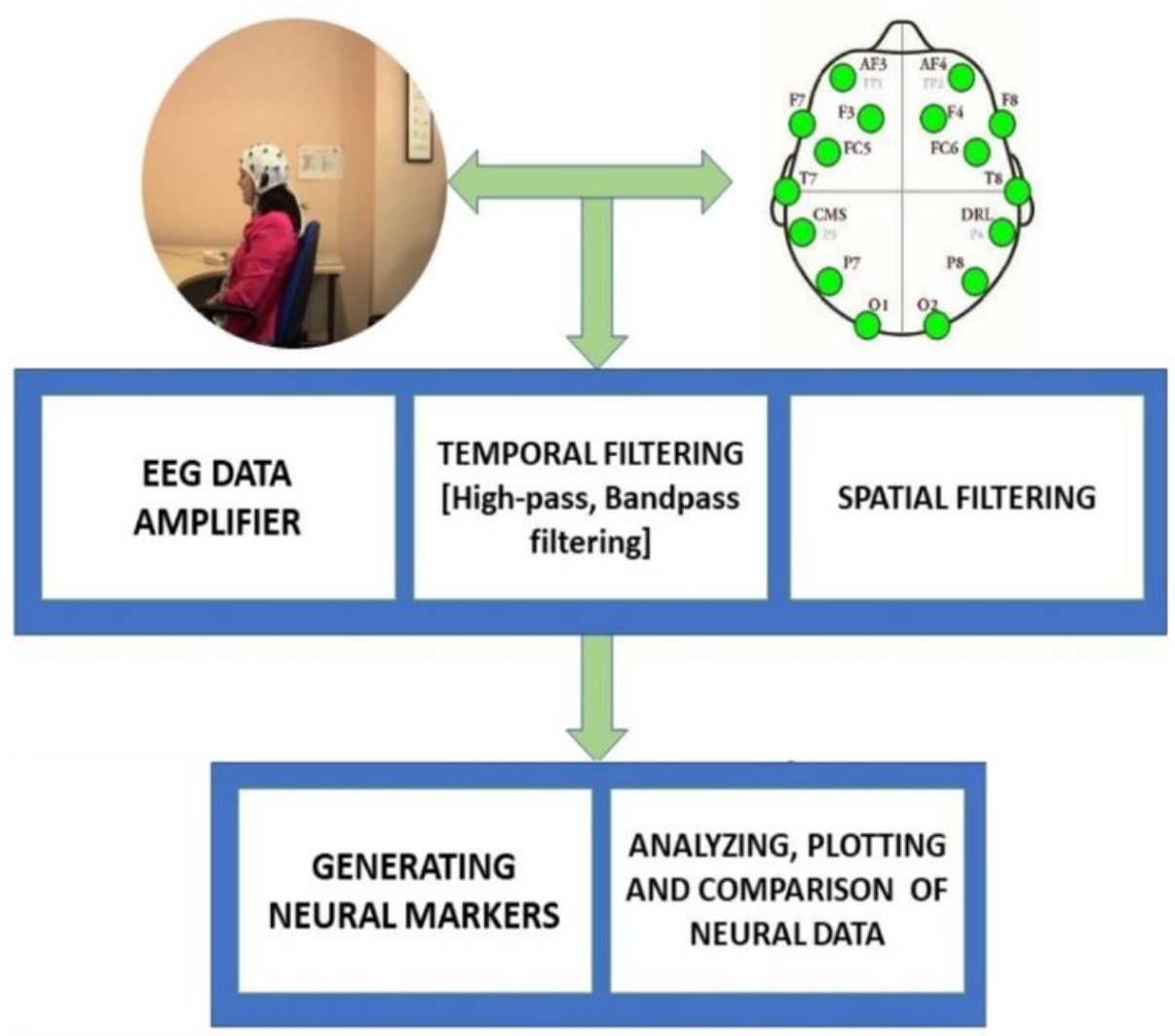

Figure 1

Graphical abstract explaining the methodology for Analyzing neural data for Media Addicts

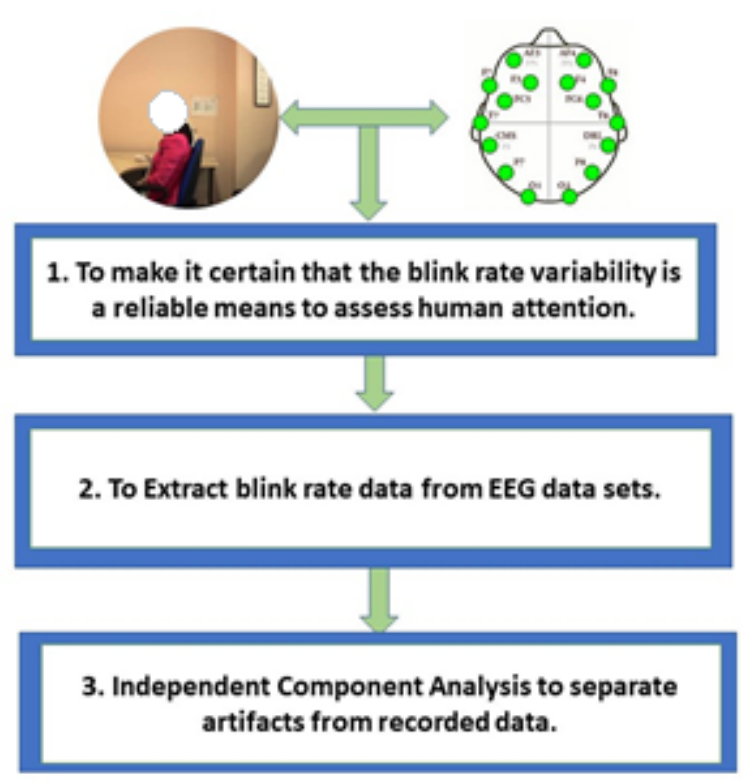

Figure 2

Graphical abstract explaining the methodology for analyzing Computer Vision Syndrome 


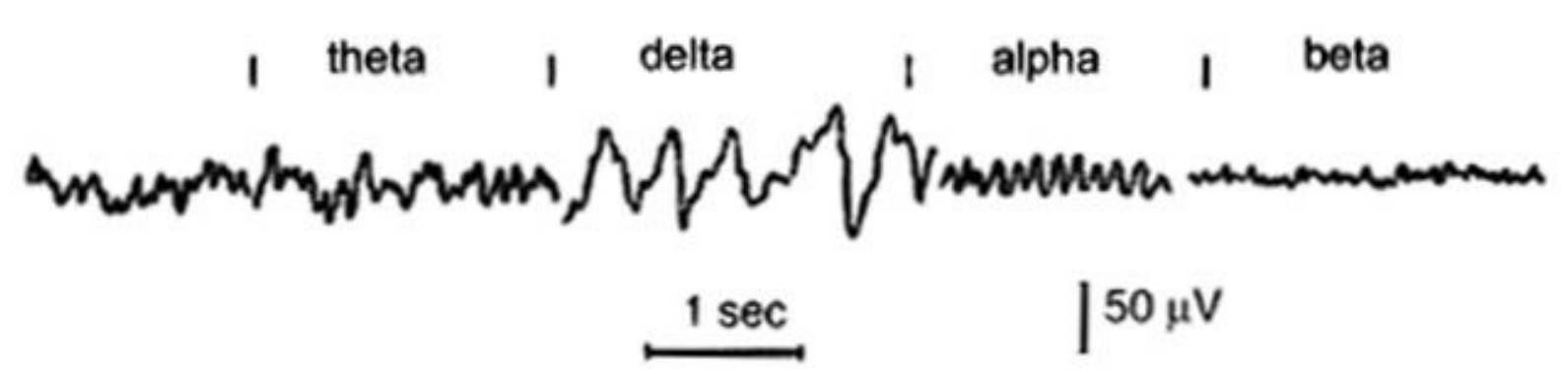

Figure 3

Different types of EEG waves. Source: www.medicine.mcgill.ca 


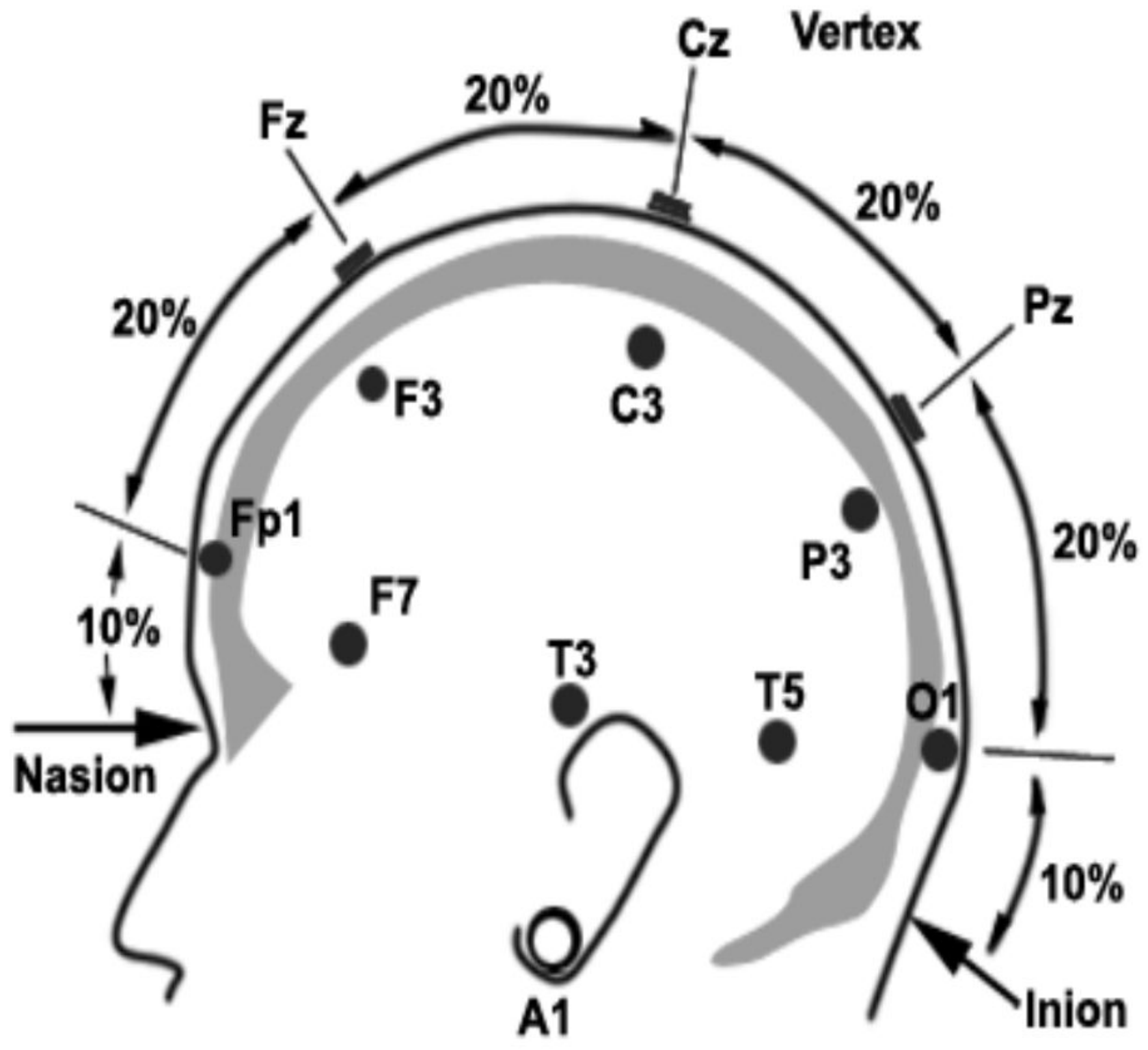

Figure 4

10/20 International electrodes positioning system 


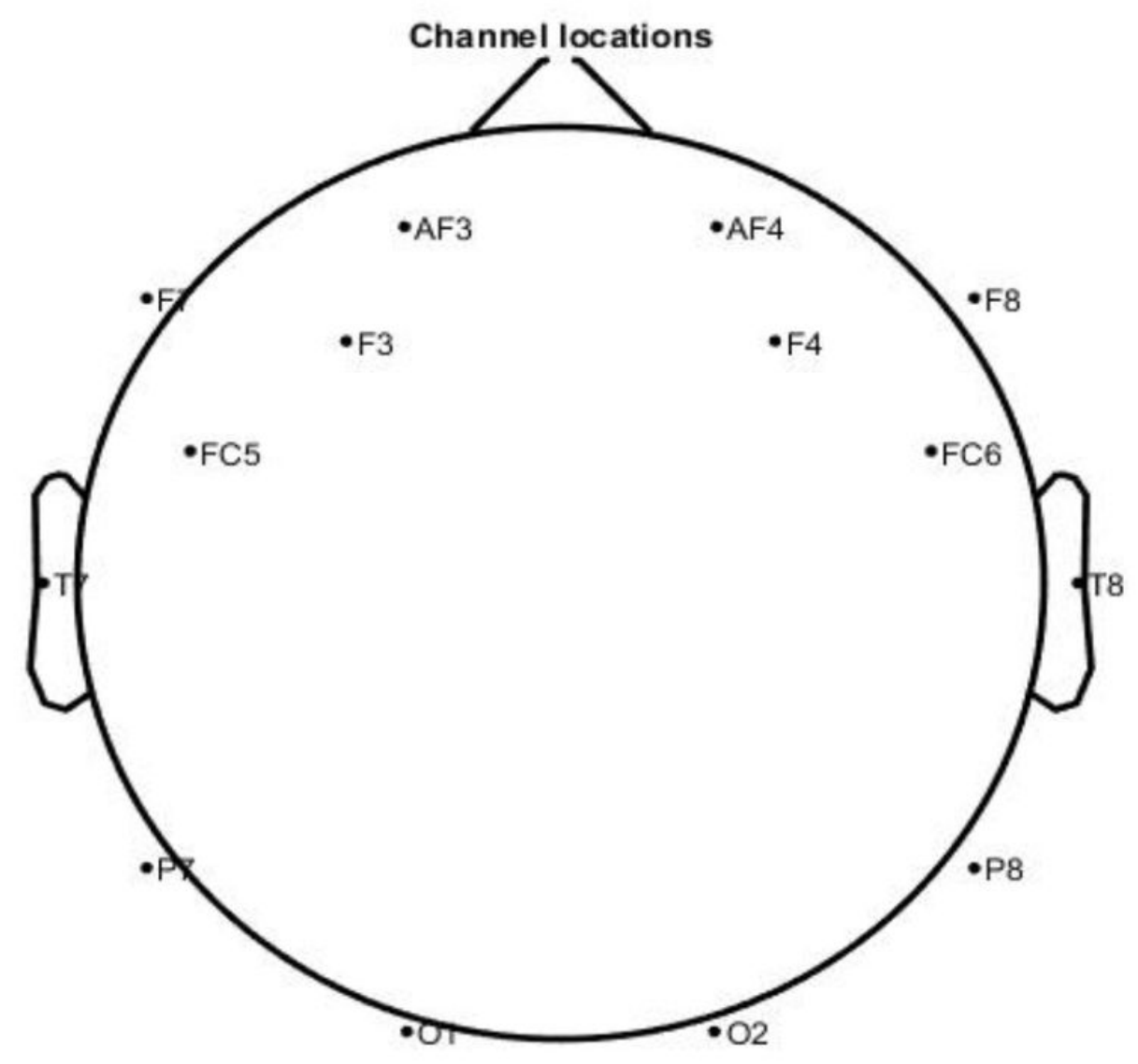

14 of 14 electrode locations shown

Figure 5

Perspective of Electrode locations placed 


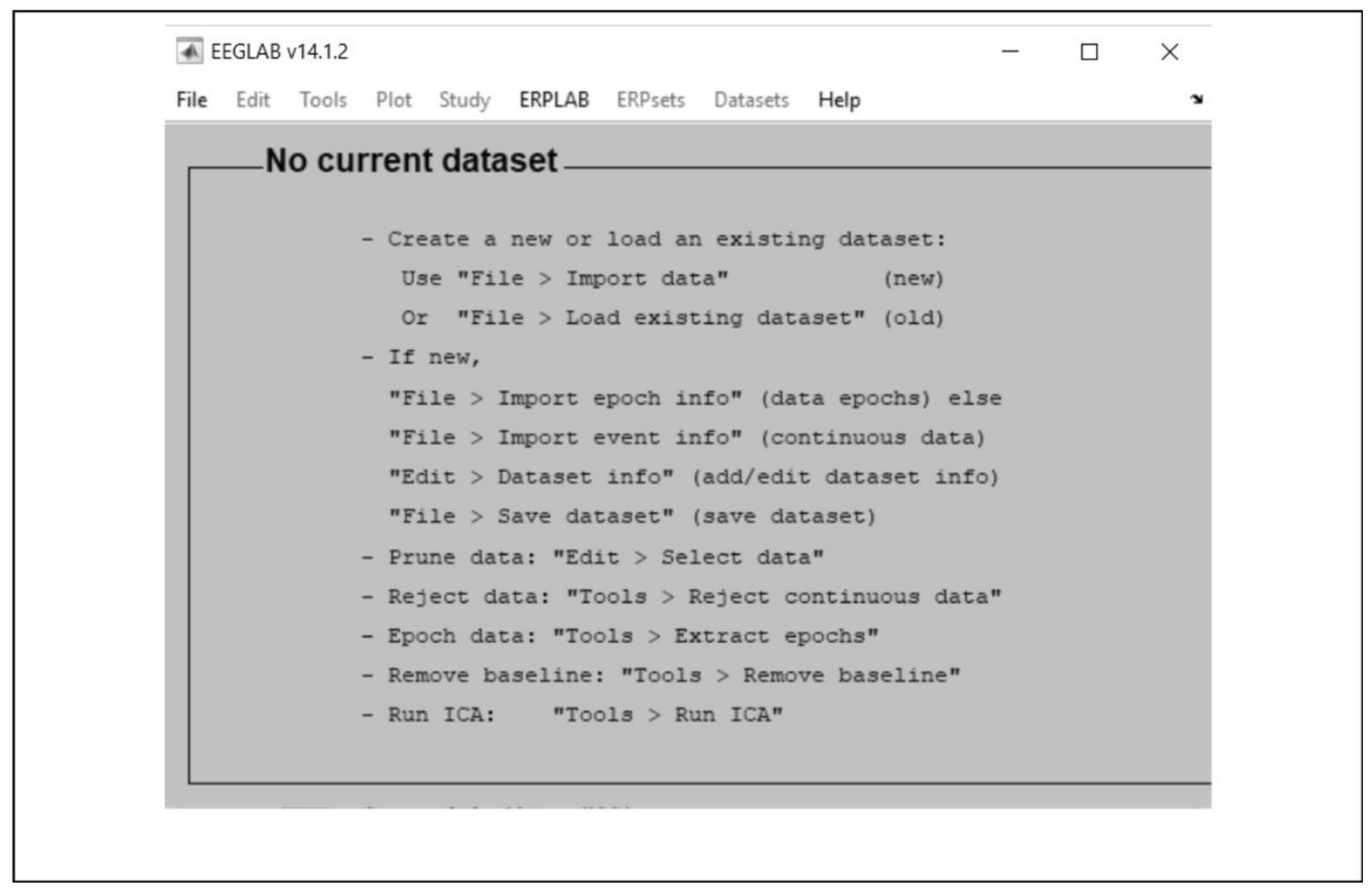

Figure 6

EEGLAB window in MATLAB

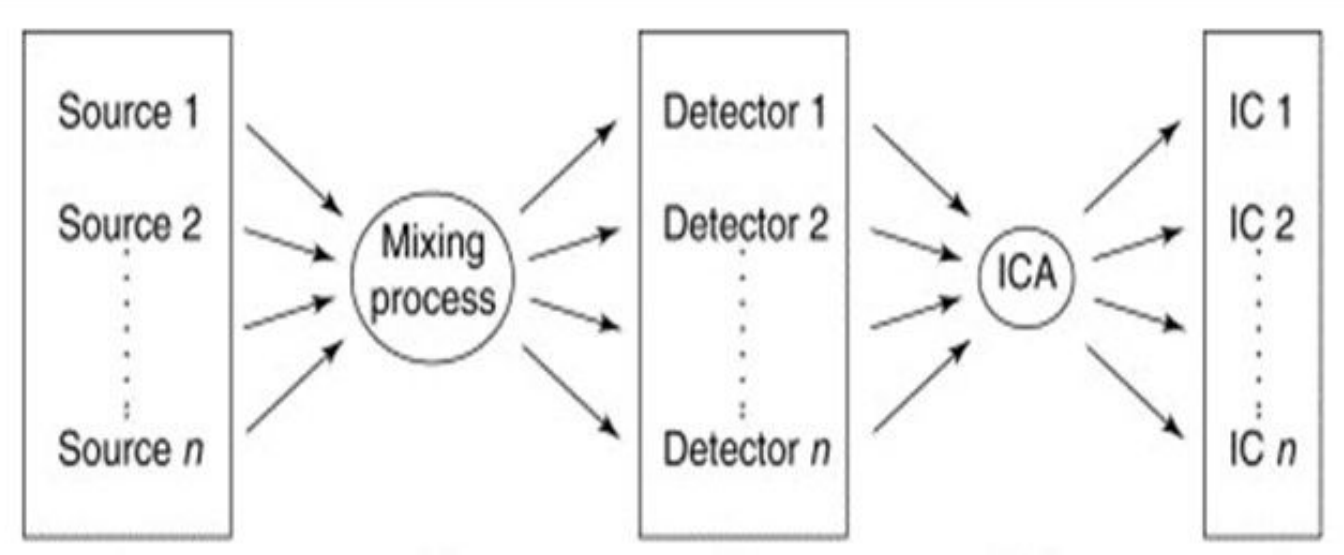

Figure 7

Fast/CA Working explained 


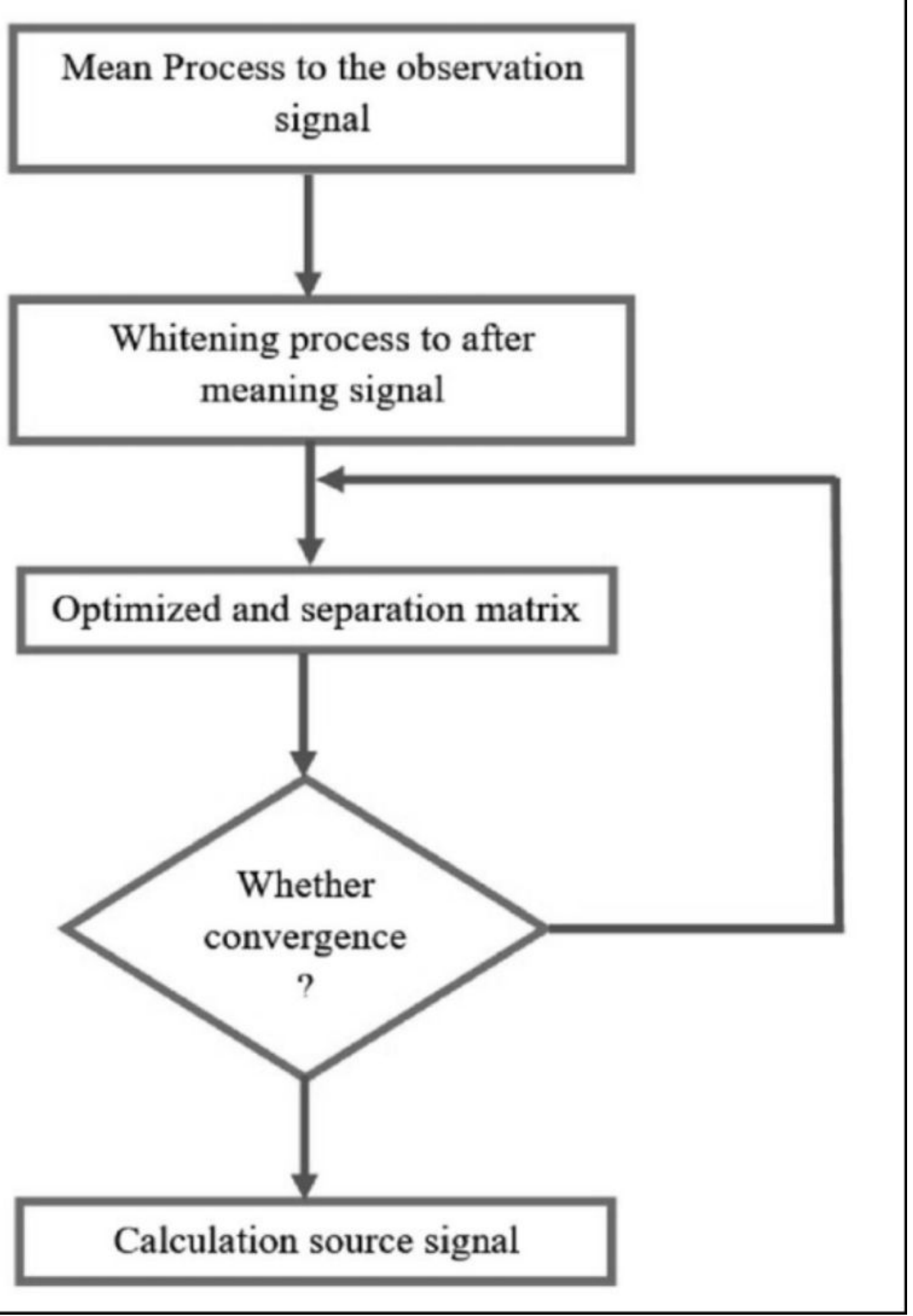

Figure 8

Flowchart for FastICA Algorithm [23] 

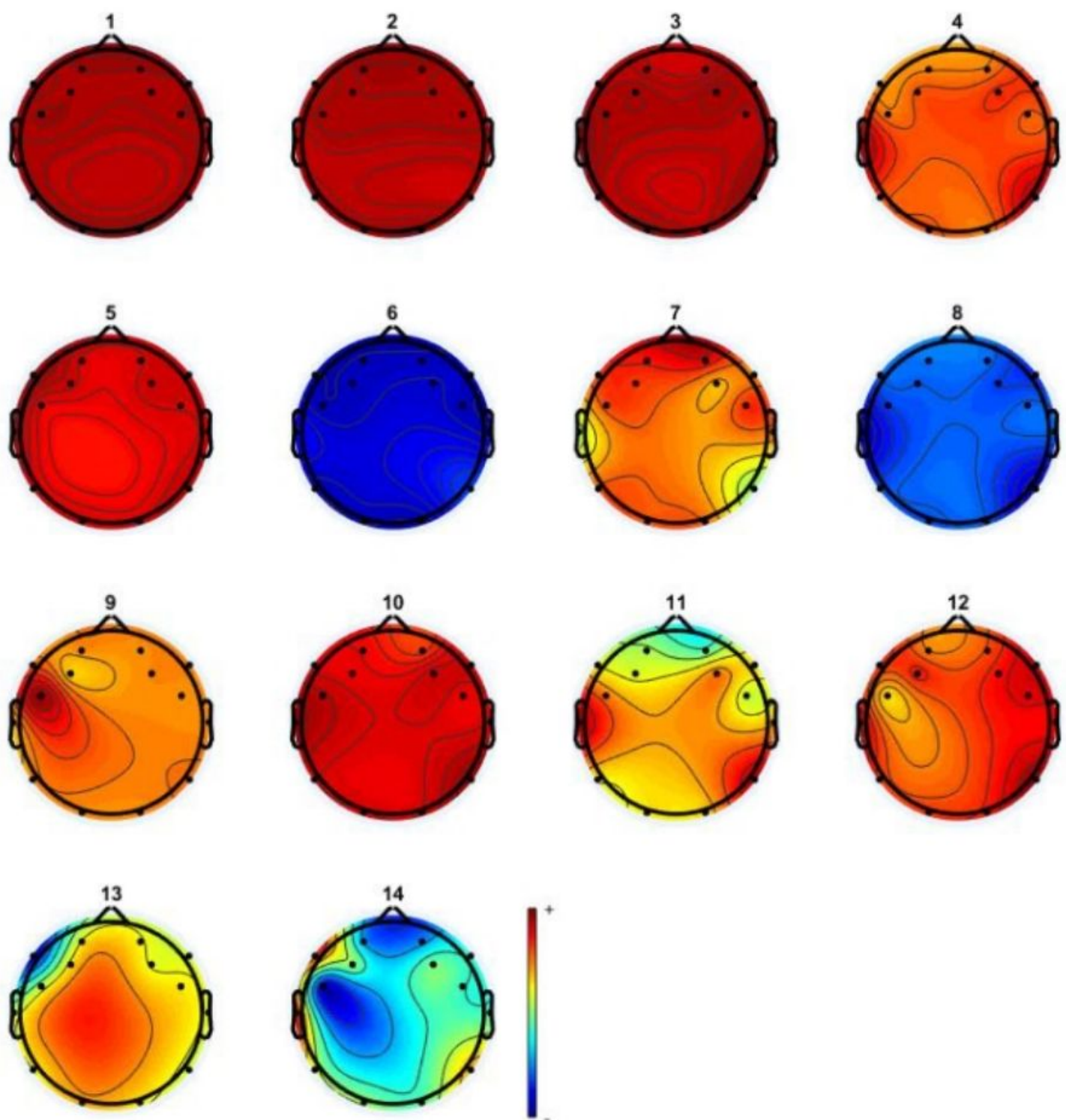

EDF Data - Component Maps in 2D

Figure 9

2D Component Maps 


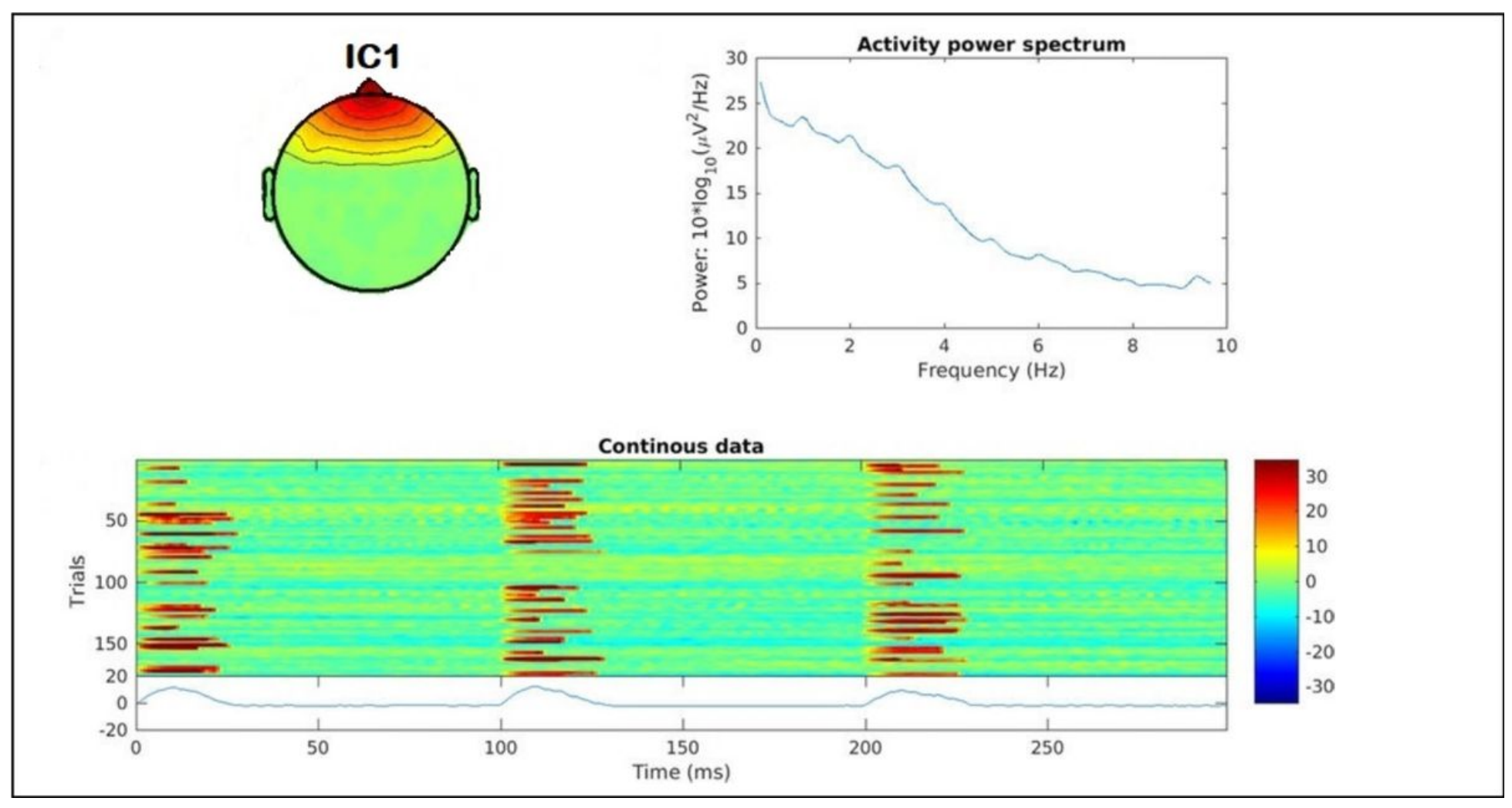

Figure 10

IC1 representing blinks in the spectrum 


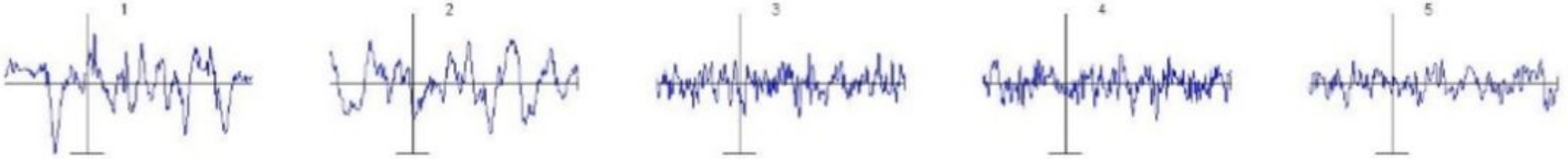
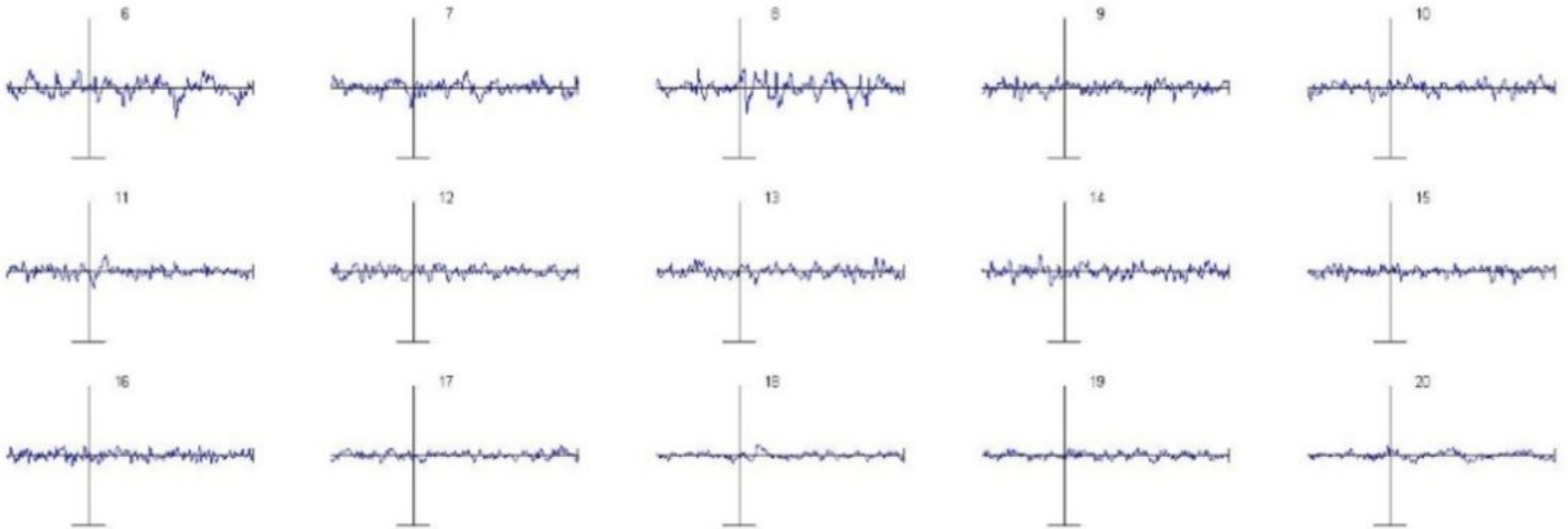

19
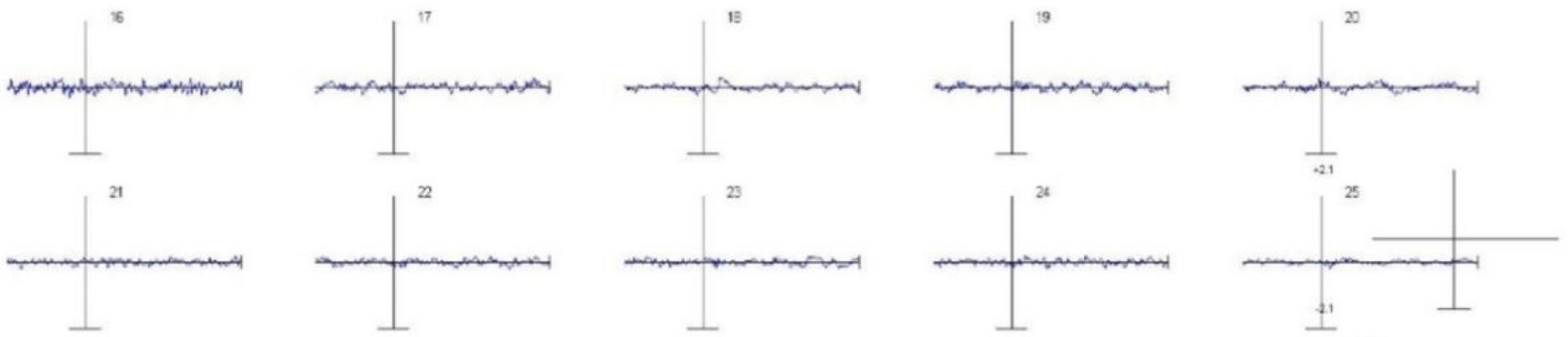

$.1 \times$

\section{Figure 11}

Individual Components with Eye blinks

\section{IC Component cross-coherence}

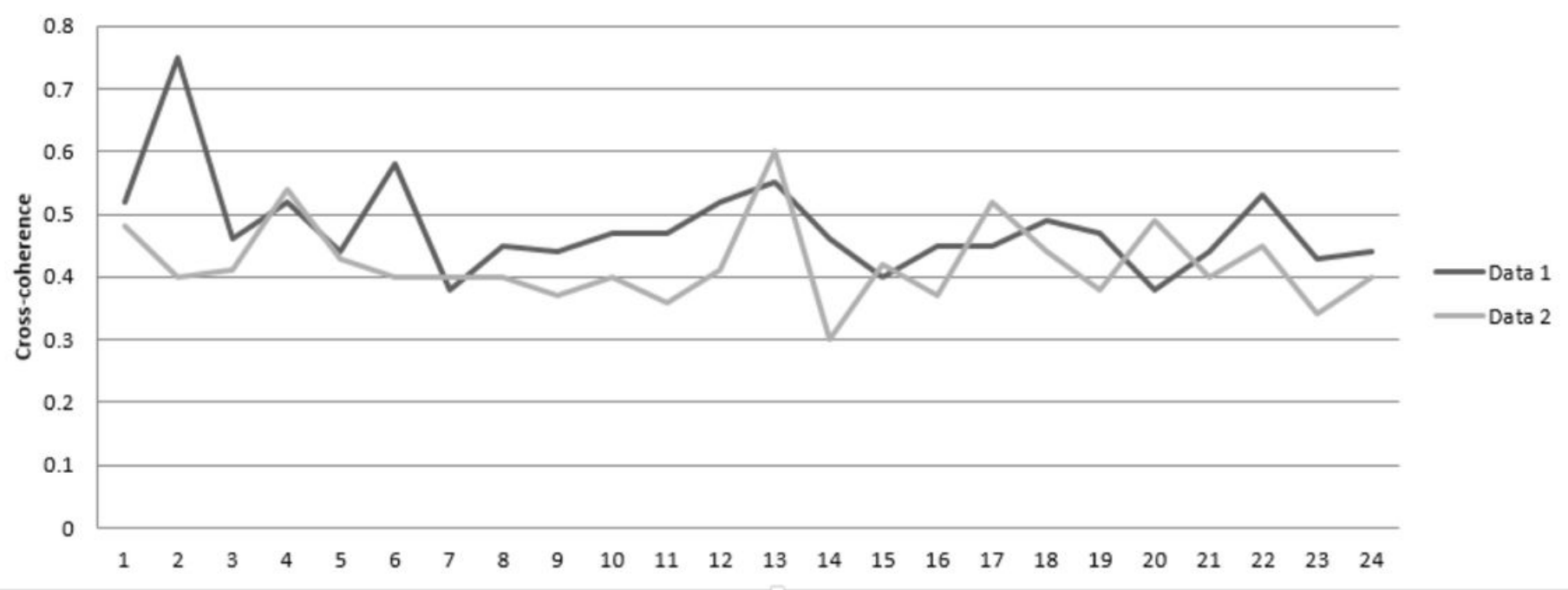

Figure 12 


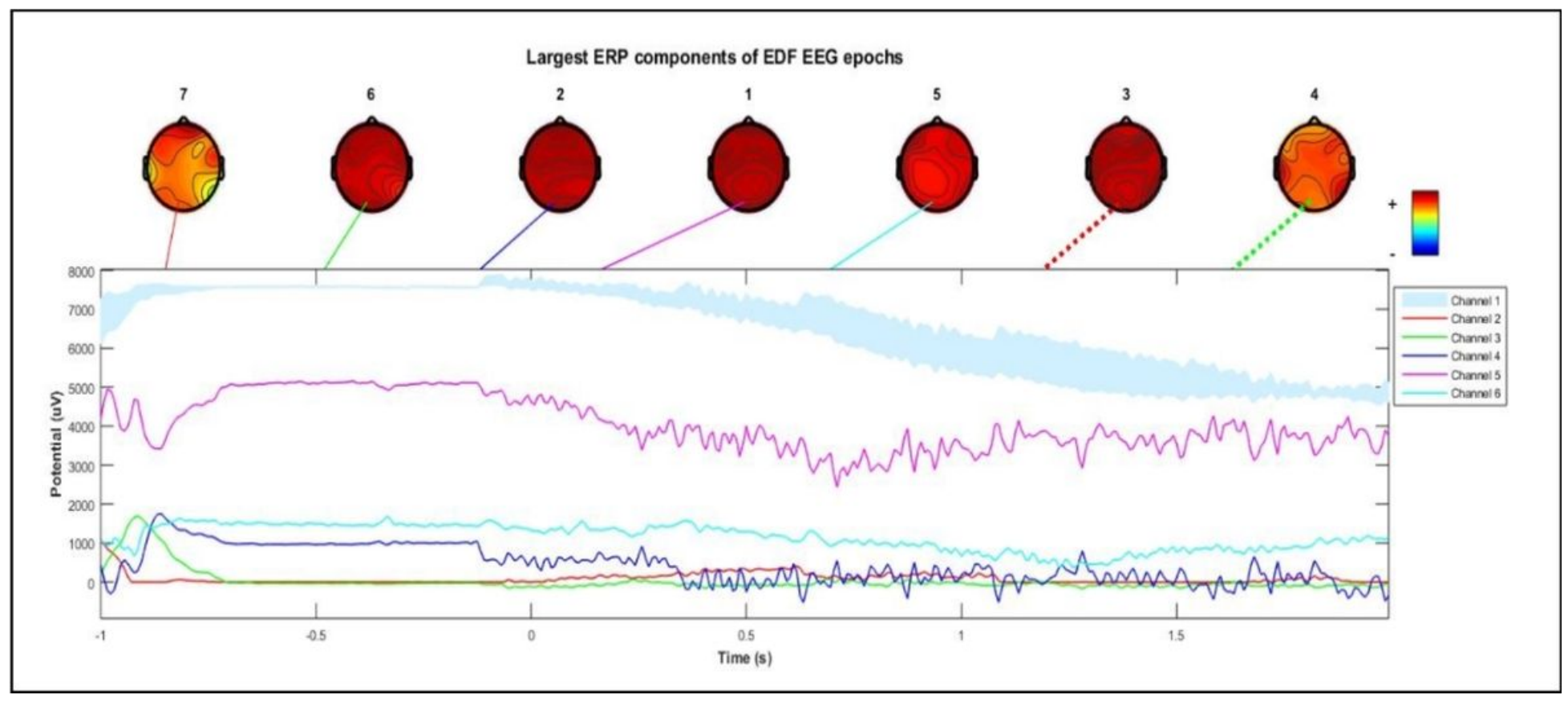

\section{Figure 13}

Identified Event-Related Potentials (ERP) in a sampled data 


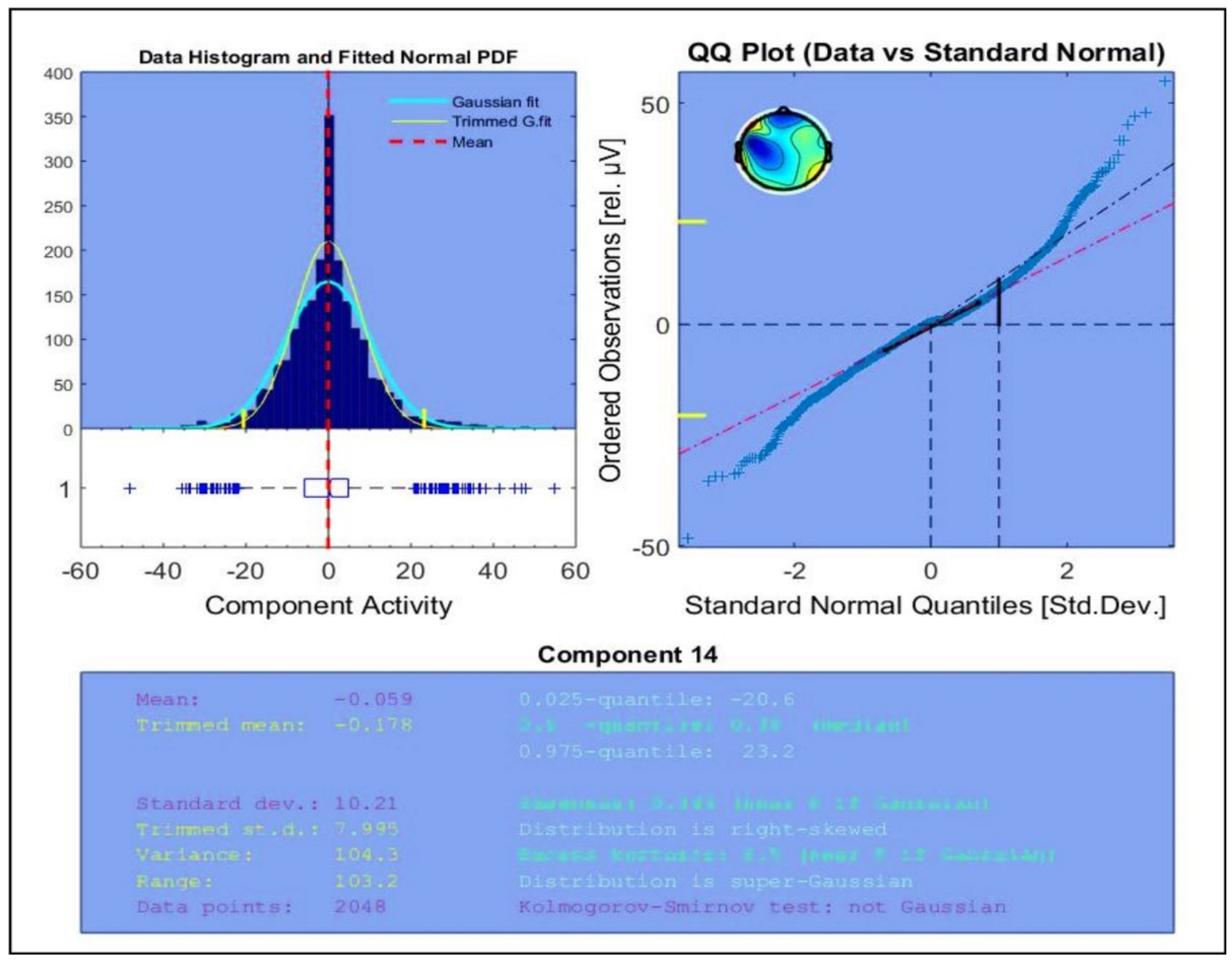

Figure 14

Component Activity IC14 and Standard Normal Quantities 


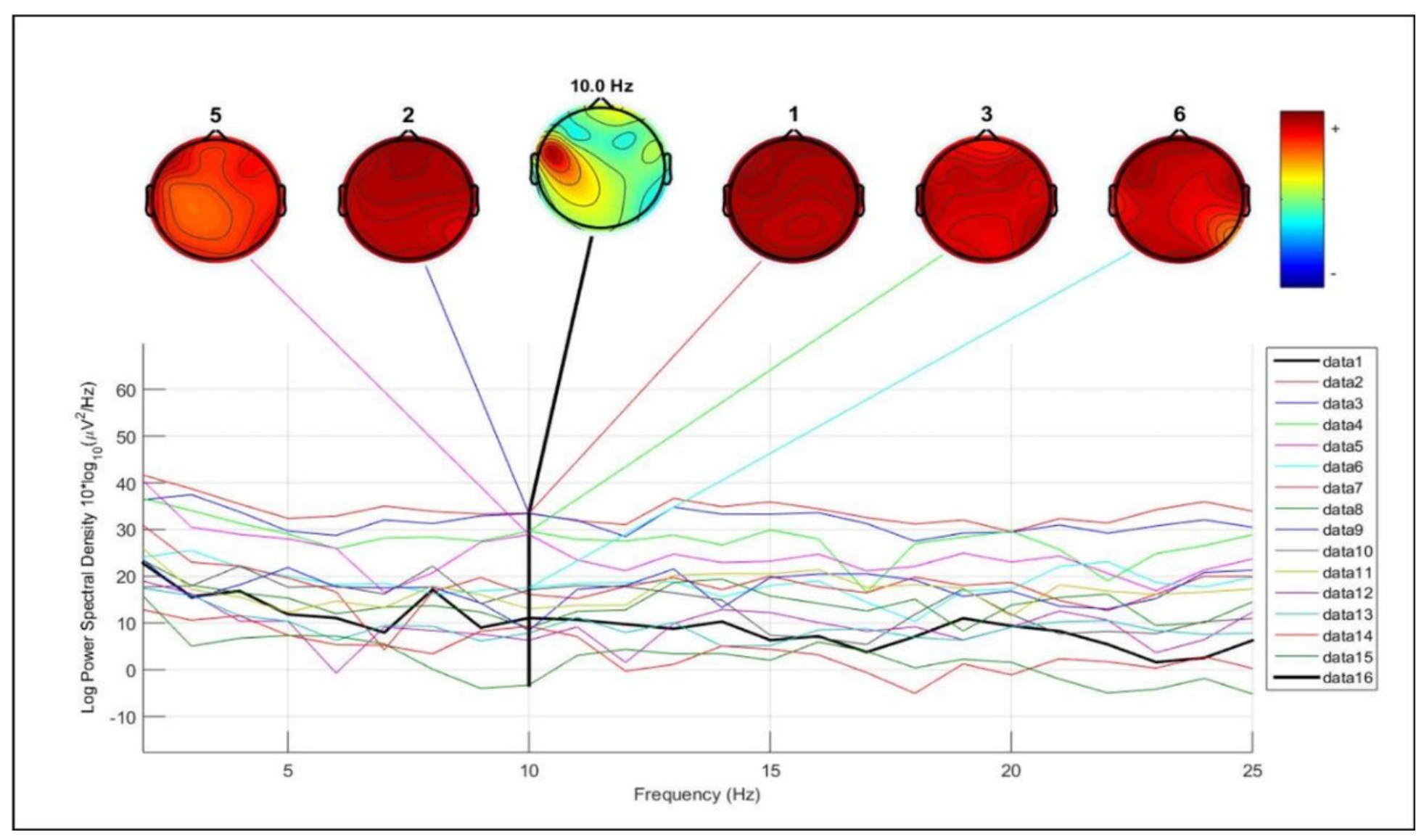

Figure 15

Power spectral density for components 

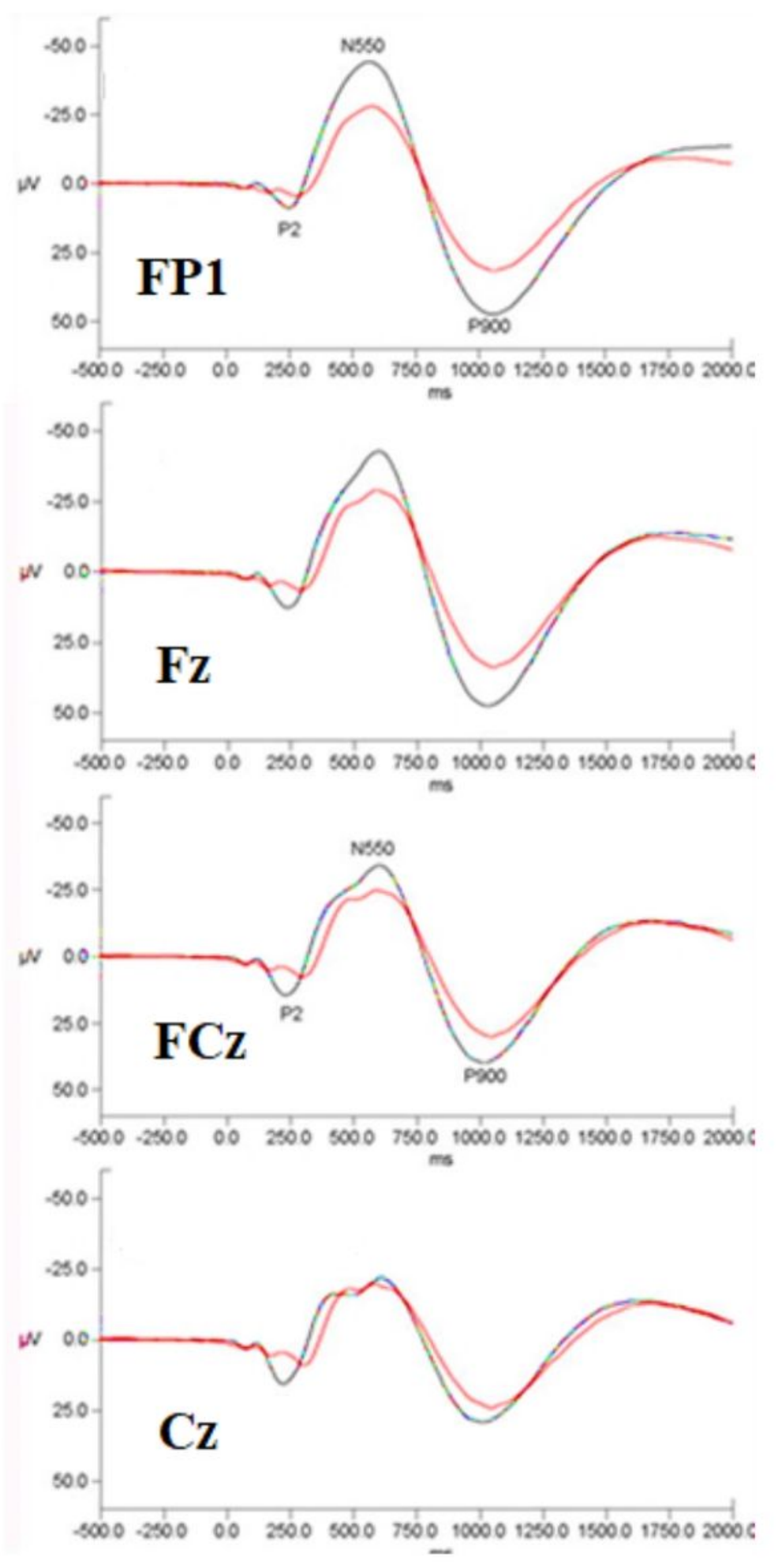

Figure 16

Grand mean evoked potential waveforms for alcoholics (red lines) and control subjects (black lines). Data drawn from (Colrain et al. 2009) [24]. 


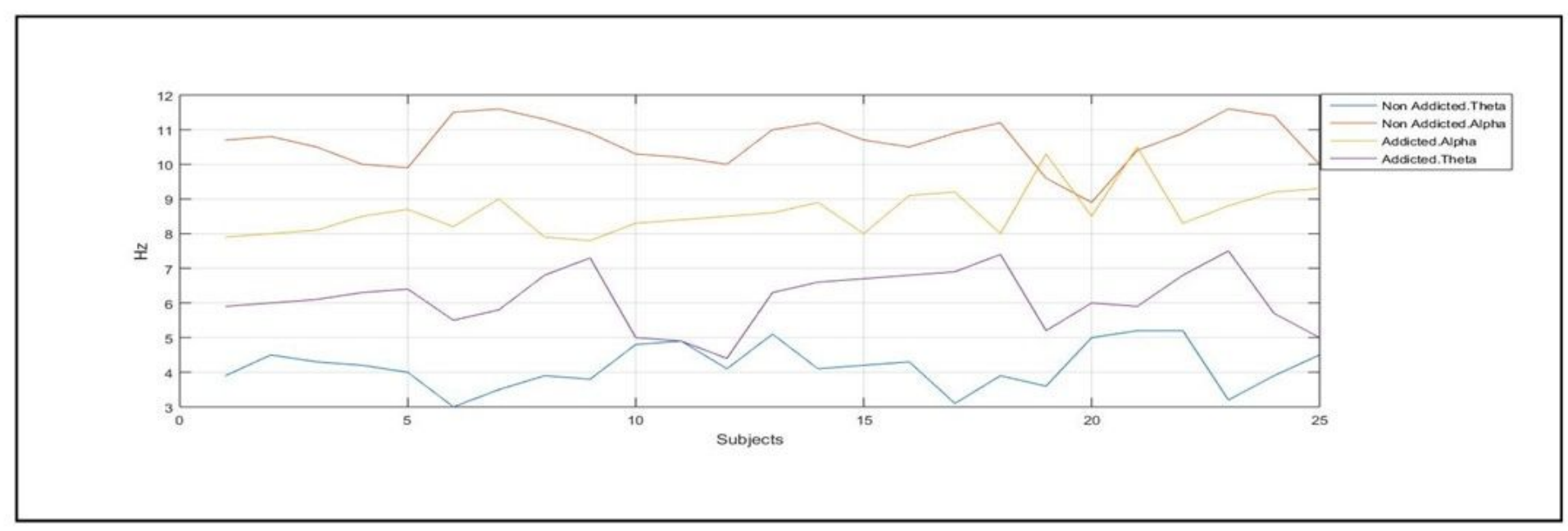

Figure 17

Overall trend of Alpha \& Theta of Media Addicted vs non-Addicted 


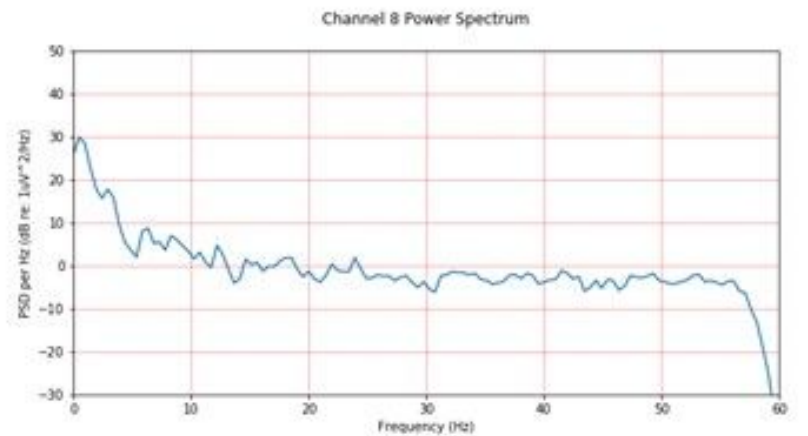

a. Power Spectrum of Channel 8

Channel 6 Power Spectrum

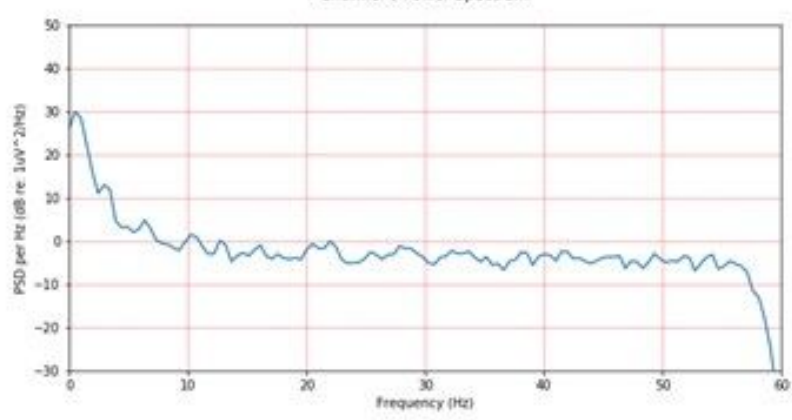

c. Power Spectrum of Channel 6

Channel 4 Power Spectrum

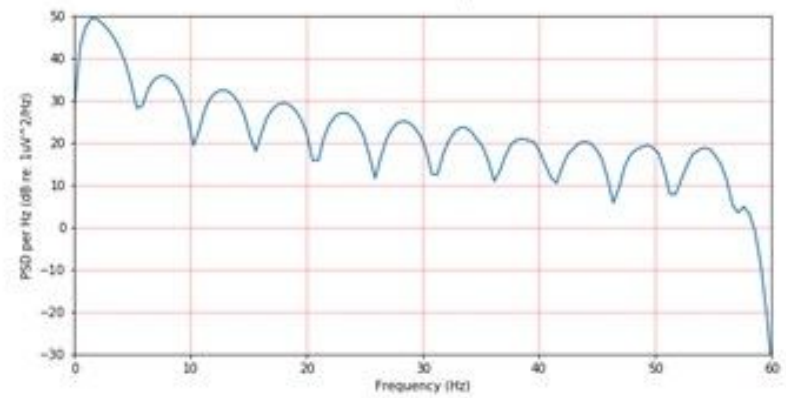

e. Power Spectrum of Channel 4

channel 2 Power Spectrum

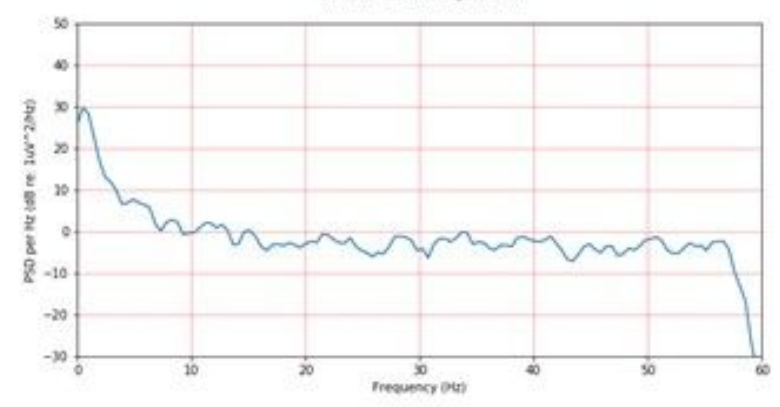

g. Power Spectrum of Channel 2

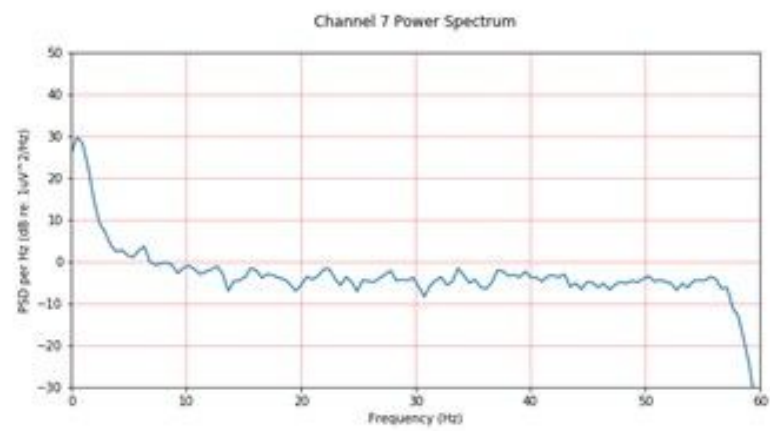

b. Power Spectrum of Channel 7

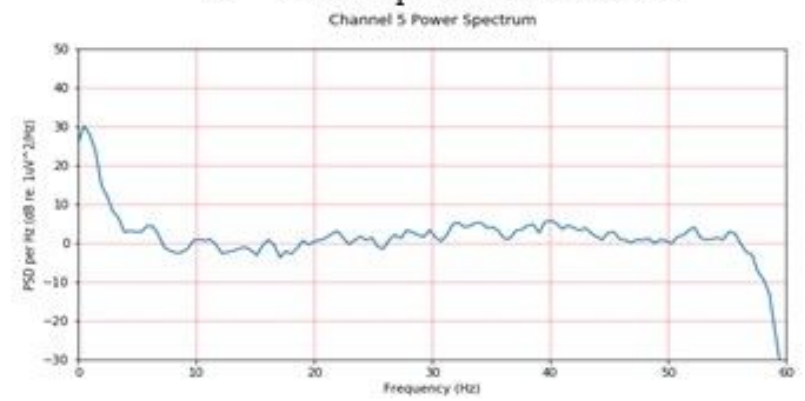

d. Power Spectrum of Channel 5 channel 3 power Spectrum

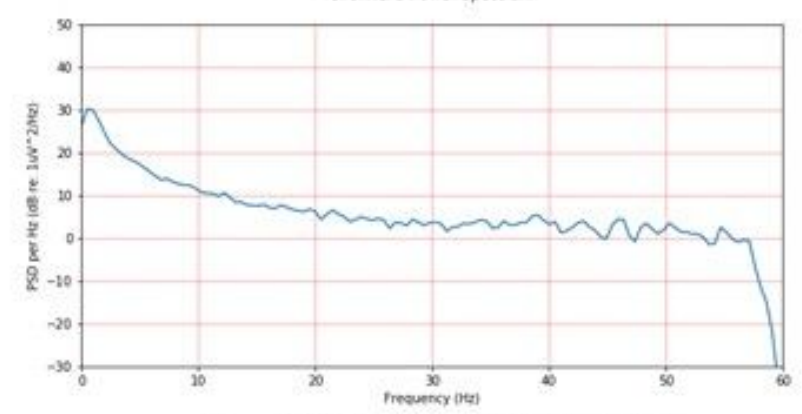

f. Power Spectrum of Channel 3 channel 1 Power Spectrum

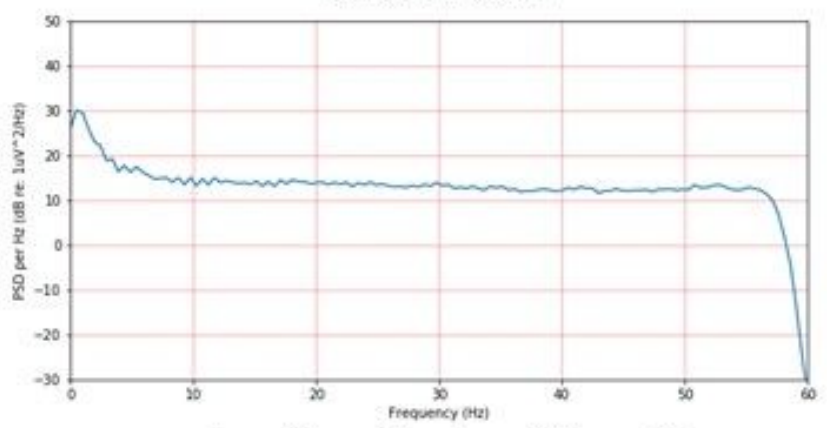

h. Power Spectrum of Channel 1

\section{Figure 18}

Power spectral analysis a-h (Channel 8 - 1) 


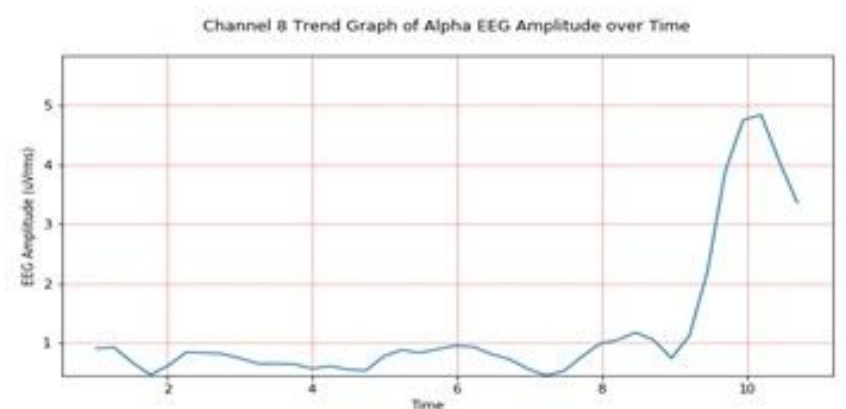

a. Trend graph Channel 8 .

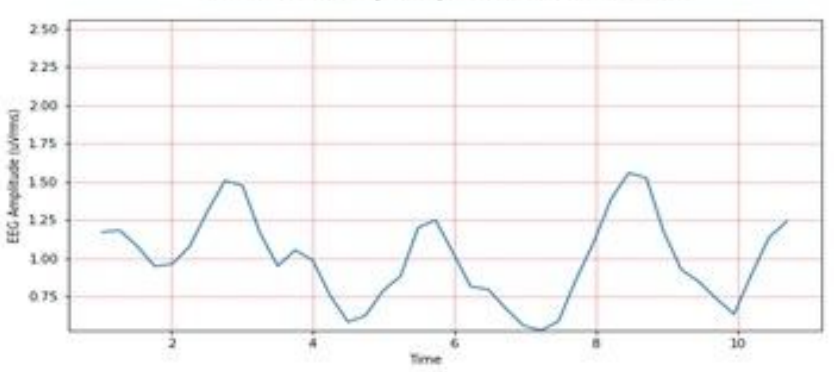

c. Trend graph Channel 6 .

Channel 4 Trend Graph of Alpha EEG Amplitude over Time

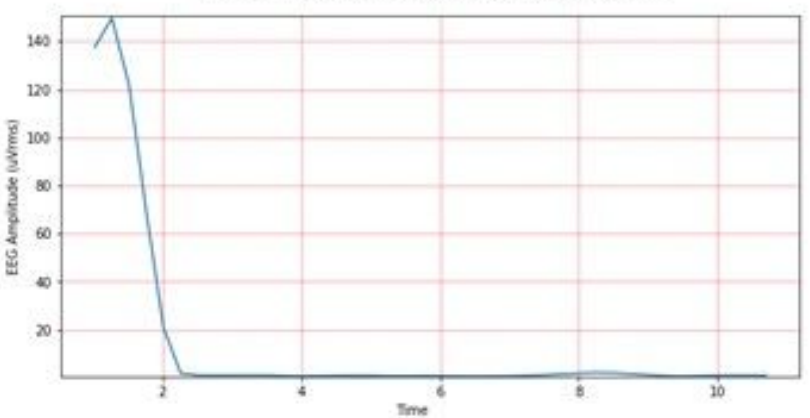

e. Trend graph Channel 4.

Channel 2 Trend Graph of Alpha EEG Ampltude over Time

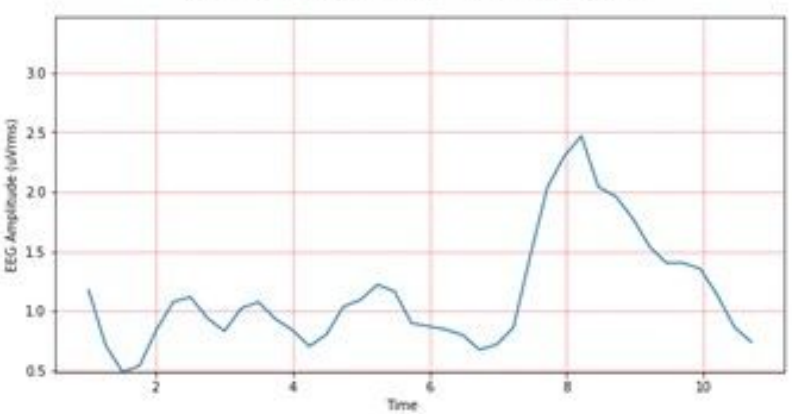

g. Trend graph Channel 2.

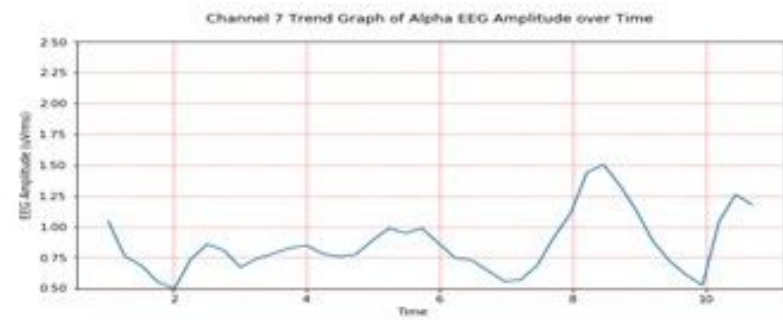

b. Trend graph Channel 7 .

Channel 5 Trend Graph of Alpha EEG Amplitude over Time

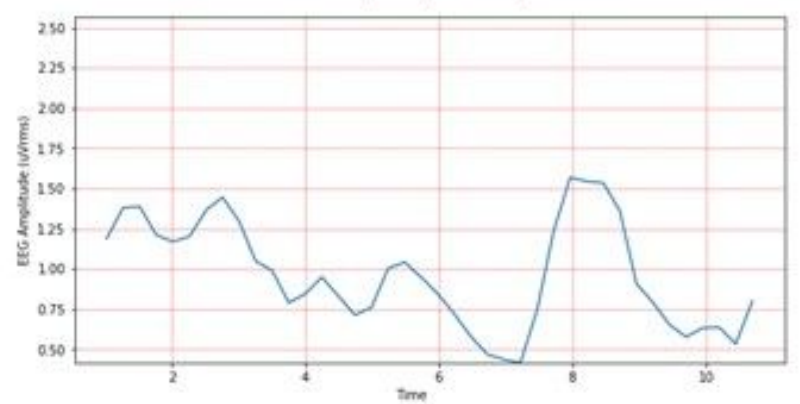

d. Trend graph Channel 5.

Channel 3 Trend Graph of Alpha EEG Amplitude over Time

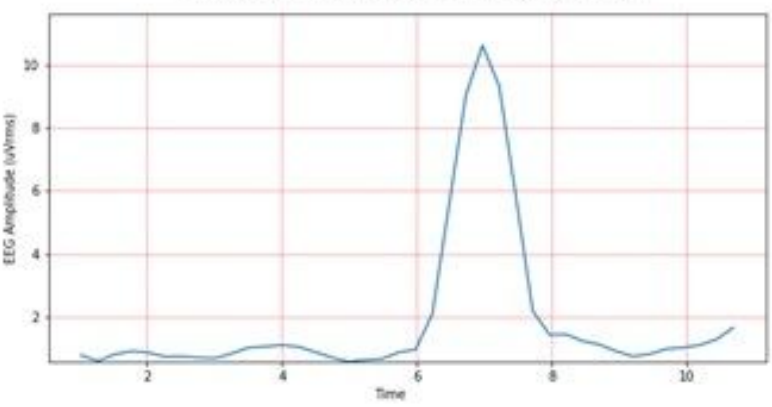

f. Trend graph Channel 4.

Channel 1 Trend Graph of Alpha EEG Amplatude ower Time

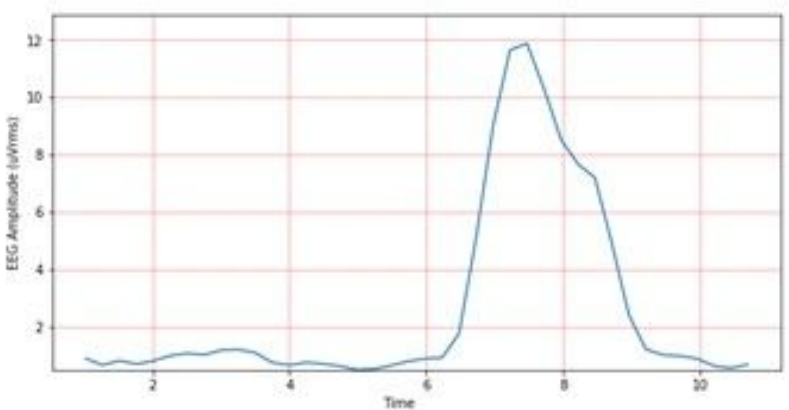

h. Trend graph Channel 1.

\section{Figure 19}

Trend Graphs a-h (Channel $8-1)$ 


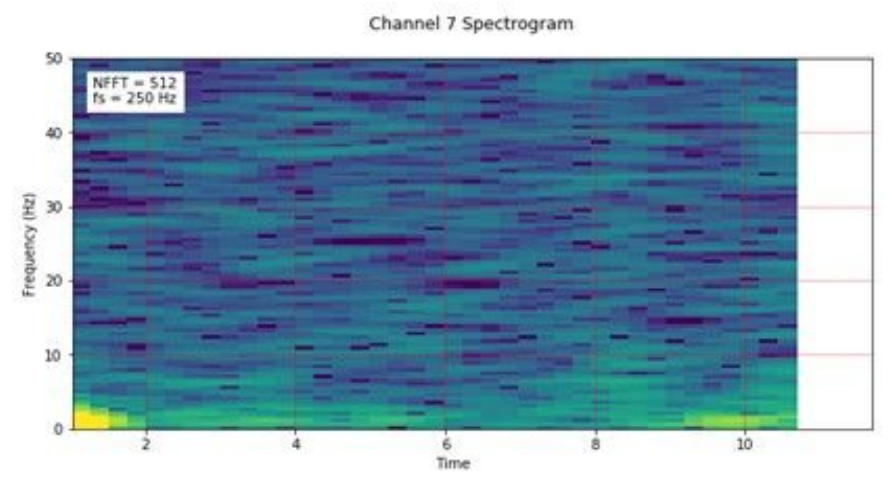

a. Spectrogram of Channel 7 Channel 5 Spectrogram

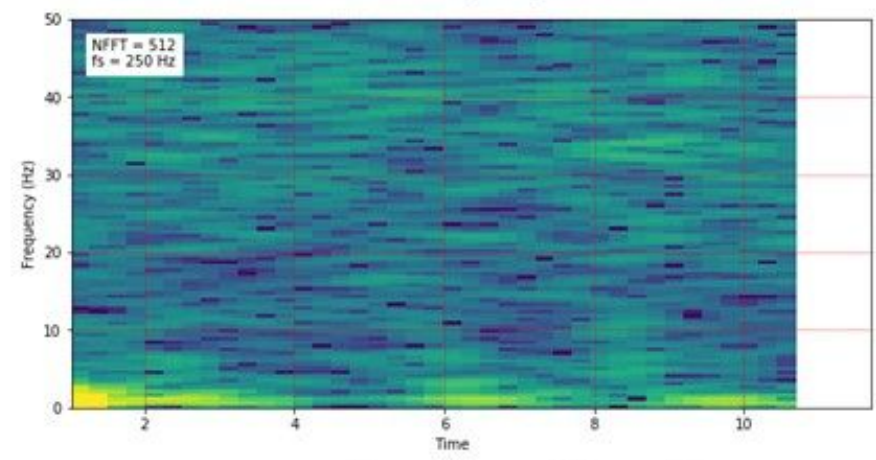

c. Spectrogram of Channel 5 Channel 3 Spectrogram

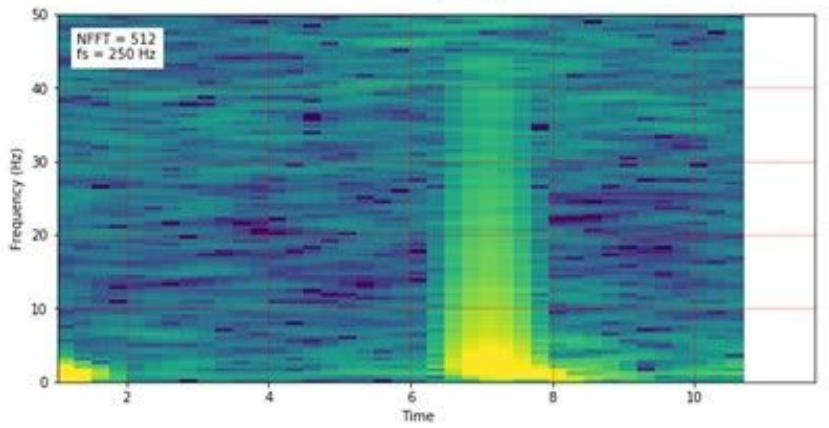

e. Spectrogram of Channel 3

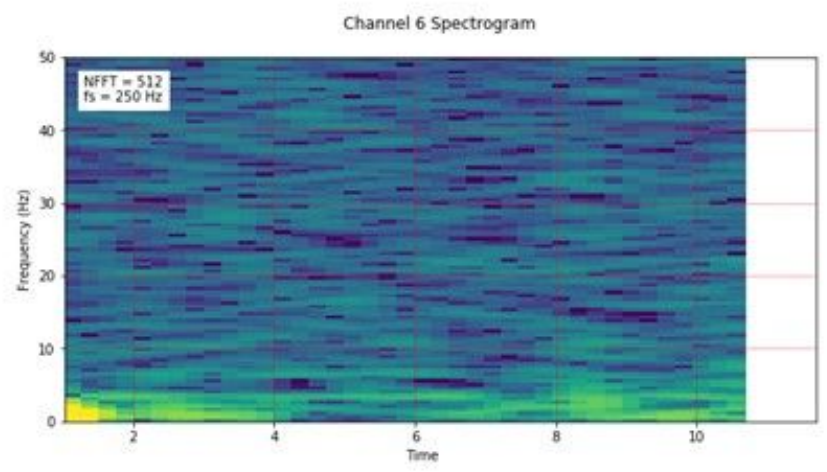

b. Spectrogram of Channel 6 channel 4 Spectrogram

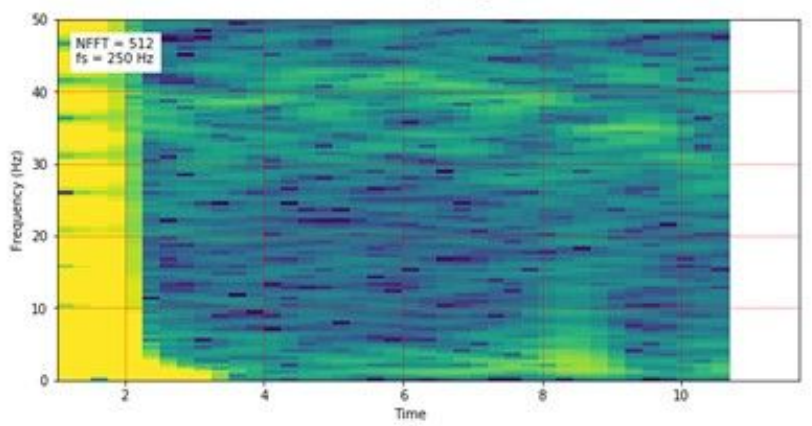

d. Spectrogram of Channel 4 Channel 1 Spectrogram

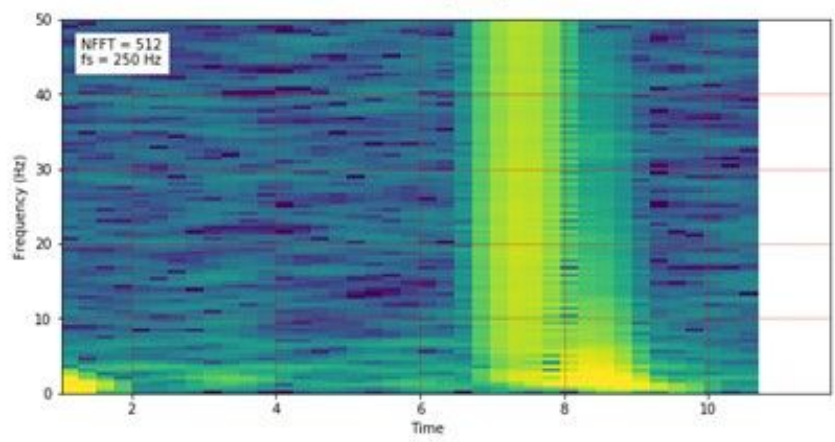

f. Spectrogram of Channel 1

Figure 20

Spectrogram of Channel 1-6 